\title{
A minimization problem for the lapse and the initial-boundary value problem for Einstein's field equations
}

\author{
Gabriel Nagy $^{1}$ and Olivier Sarbach ${ }^{1,2}$ \\ ${ }^{1}$ Department of Mathematics, University of California at San Diego, 9500 Gilman Drive, \\ La Jolla, CA 92093-0112, USA \\ ${ }^{2}$ Theoretical Astrophysics 130-33, California Institute of Technology, Pasadena, CA 91125, USA
}

Received 26 January 2006, in final form 3 April 2006

Published 27 July 2006

Online at stacks.iop.org/CQG/23/S477

\begin{abstract}
We discuss the initial-boundary value problem of general relativity. Previous considerations for a toy model problem in electrodynamics motivate the introduction of a variational principle for the lapse with several attractive properties. In particular, we show that the resulting elliptic gauge condition for the lapse together with a suitable condition for the shift and constraintpreserving boundary conditions controlling the Weyl scalar $\Psi_{0}$ yields a priori estimates on the metric, connection and curvature fields. These estimates are expected to be useful for obtaining a well-posed initial-boundary value problem for metric formulations of Einstein's field equations which are commonly used in numerical relativity. To present a simple and explicit example, we consider the $3+1$ decomposition introduced by York of the field equations on a cubic domain with two periodic directions and prove in the weak field limit that our gauge condition for the lapse, an a priori specified shift vector and our boundary conditions lead to a well-posed problem. The method discussed here is quite general and should also yield well-posed problems for different ways of writing the evolution equations, including first-order symmetric hyperbolic or mixed first-order second-order formulations. Well-posed initial-boundary value formulations for the linearization about arbitrary stationary configurations will be presented elsewhere.
\end{abstract}

PACS numbers: 04.20.Ex, 04.20.-q, 04.25.Dm

\section{Introduction}

A common approach to numerical relativity is to find solutions of Einstein's field equations on a cylinder of the form $I \times \Sigma$, where $I=[0, T]$ is a time interval and $\Sigma$ is a three-dimensional compact manifold with smooth boundary $\partial \Sigma$. The initial-boundary value problem (IBVP) 
consists in finding solutions with specified (initial) data on the 'bottom' of this cylinder (i.e. on $\{0\} \times \Sigma)$ and boundary data on the set $\mathcal{T} \equiv I \times \partial \Sigma$. The exact form of the boundary data depends on the nature of $\mathcal{T}$; in this paper we consider the case where $\mathcal{T}$ is timelike. This excludes the case of inner black hole excision boundary conditions where $\mathcal{T}$ is spacelike or null, and the case where spacetime is compactified in such a way that $\mathcal{T}$ is a null surface.

For timelike $\mathcal{T}$, boundary conditions must be specified with care. First, they should be stable in the mathematical sense of well posedness of the resulting IBVP which means that one can guarantee local in time existence of solutions which lie in an appropriate normed function space and which depend uniquely and continuously on the initial and boundary data. Second, the boundary conditions should be constraint-preserving which means that they must guarantee that a solution to the IBVP with constraint-satisfying initial data automatically satisfies the constraints everywhere on $I \times \Sigma$. Third, the boundary data should enable control over the gravitational flux through the boundary, at least in some approximate sense. This is a difficult question since in general relativity there are no meaningful local expressions for quantities representing the energy density of the gravitational field. This is related to the fact that locally, the gravitational field can always be transformed away. Finally, it would be desirable if boundary conditions could be specified in such a way to control part of the geometry of $\mathcal{T}$.

A well-posed IBVP of Einstein's vacuum field equations addressing all the above points has been presented in [1]. This work is based on the use of tetrad fields in a gauge adapted to the boundary $\mathcal{T}$ and casting the evolution equations into a first-order symmetric hyperbolic system for the tetrad fields, the connection coefficients and the components of the Weyl tensor. Furthermore, the evolution equations in [1] imply that the constraint propagation system, describing the evolution of the constraint fields, has the form of a hyperbolic system which on $\mathcal{T}$ contains only derivatives tangential to $\mathcal{T}$. As a consequence, the satisfaction of the constraintpreserving property is automatic. The only fields with non-trivial characteristic speeds at the boundary are the Weyl scalars $\Psi_{0}$ and $\Psi_{4}$ that can be interpreted as describing in- and outgoing gravitational radiation, at least in some approximate sense. (Note that our definition of $\Psi_{0}$ and $\Psi_{4}$ differs from that in [1].) By specifying data to $\Psi_{0}$ or a suitable combination of $\Psi_{0}$ and $\Psi_{4}$, the work in [1] obtains a well-posed IBVP for Einstein's vacuum field equations by applying standard theorems on symmetric hyperbolic equations with maximally dissipative boundary conditions [2-4].

On the other hand, most numerical codes are based on formulations of Einstein's equations which use metric variables and not tetrad fields. For such formulations, the problem of obtaining a well-posed IBVP is open. One of the major obstacles stems from the fact that compatibility with the constraints yields boundary conditions which have the form of differential equations on the boundary for which no standard mathematical theorems are known. Despite several partial results [5-10], the derivation of necessary conditions for well posedness $[9,11-14]$ and numerical experiments [5-7, 13, 15-20], a full understanding of the IBVP for metric formulations has not yet been achieved.

In this paper, we reconsider the IBVP for Einstein's field equations by combining the ideas of [1] with new ideas developed in [21] for a model problem in electrodynamics. These new ideas, which are reviewed in section 2, are based on separate estimates for the constraint fields, the curvature fields and the imposition of an appropriate gauge condition which allows an estimate for the remaining, gauge-dependent fields. Boundary conditions on the constraint and curvature fields are an essential part in obtaining these estimates. In the electromagnetic case, the curvature fields are the electric and magnetic fields which are gauge-invariant. In general relativity, the curvature field is described by the Riemann tensor which can be decomposed into the Ricci tensor and the Weyl tensor. Motivated by the model problem in [21] we consider a gauge condition for the lapse that is obtained by minimizing a simple functional representing 
the norm squared of the time derivative of the extrinsic curvature. The minimization principle, which yields a fourth-order elliptic boundary value problem for the lapse, possesses several useful properties. One of them is that the functional is positive semi-definite and zero if, and only if, the components of the extrinsic curvature are time-independent. Therefore, the gauge condition should be well adapted to stationary configurations or small deviations thereof. In section 2.6 we summarize the boundary conditions, the gauge conditions and the evolution system considered in this paper and write down the resulting IBVP. In section 3 we examine the variational principle for the lapse in the weak field limit, establish its well posedness in theorem 1 and give a physical interpretation for it as a gauge-fixing procedure for the extrinsic curvature. In lemma 1 we also derive some key estimates for the extrinsic curvature which are valid in this gauge.

The main result of this paper is derived in section 4 . There, we consider the $3+1$ decomposition of the Einstein vacuum field equations as described in [22] in the limit of linearizations about flat spacetime and couple them to the fourth-order elliptic gauge condition for the lapse. We prove in theorem 3 that this elliptic-hyperbolic system, together with zero shift and constraint-preserving boundary conditions controlling a suitable combination of the Weyl scalars $\Psi_{0}$ and $\Psi_{4}$ leads to a well-posed IBVP. The proof is based on casting the evolution system into an abstract Cauchy problem on an appropriate Hilbert space and verifying that the evolution vector field generates a strongly continuous semigroup. Possible generalizations to other formulations of the linearized Einstein equations and future work are discussed in the conclusions, section 5. Appendix A summarizes standard results from the Fredholm theory used in the discussion of the gauge condition for the lapse, and appendix B contains technical proofs of some of the statements made in section 4.

\section{Main ideas}

In this section we sketch some ideas for obtaining a priori estimates in the IBVP of Einstein's field equations. We start in section 2.1 by reviewing the electromagnetic toy model problem analysed in [21] and showing that the gauge condition used in that work can be obtained by variation of a simple functional. In section 2.2, we decompose Einstein's field equations into evolution and constraint equations. Next, in section 2.3 , the constraint propagation system describing the evolution of the constraint fields is derived and a boundary condition which yields estimates for these fields is constructed. In section 2.4 we discuss how estimates for the curvature fields can be obtained. The curvature tensor can be decomposed into the Ricci tensor and the Weyl tensor. The Ricci tensor can be estimated through Einstein's equations provided suitable estimates on the matter fields are available. Therefore, it remains to estimate the Weyl tensor. In order to do so, we analyse its propagation and explain the idea for constructing boundary conditions and deriving estimates for the Weyl tensor following the lines of [1]. Next, in section 2.5, the first and second fundamental forms are estimated. For this, an appropriate gauge condition for the lapse is needed. Such a condition will be derived by introducing a functional in analogy with the electromagnetic case. Finally, the resulting IBVP for Einstein's vacuum field equations is summarized in section 2.6. The well posedness of this problem in the weak field limit is proven in the subsequent sections.

In the following, we assume that $\Sigma$ is an open bounded subset of $\mathbb{R}^{3}$ with $C^{\infty}$ boundary $\partial \Sigma$. The spacetime metric on $[0, T] \times \Sigma$ has signature $(-,+,+,+)$ and is denoted by $\mathbf{g}$ and the unique metric-compatible, torsion-free connection by $\nabla$. The induced 3-metric and connection on $\Sigma$ are denoted by $\mathbf{h}$ and $D$, respectively. The indices $a, b, c, d, e, f$ denote spacetime indices running from 0 to 3 while $i, j, k, l$ denote spatial indices running from 1 to 3 . We shall use the notation $\hat{=}$ for equalities holding on $\partial \Sigma$ or on $[0, T] \times \partial \Sigma$. The unit 
outward normal 1-form to $\partial \Sigma$ is denoted by $N_{i}$ and $\gamma_{i}{ }^{j} \hat{=} \delta_{i}{ }^{j}-N_{i} N^{j}$ is the projector onto the tangent space of $\partial \Sigma$.

\subsection{The electromagnetic case}

Maxwell's equations can be written as a first-order system for the magnetic potential $A_{j}$, the electric field $E_{j}$ and the derivatives of the magnetic potential, $W_{i j}=D_{i} A_{j}$. In the source-free case, this system is

$$
\begin{aligned}
& \partial_{t} A_{j}=E_{j}+D_{j} \phi, \\
& \partial_{t} E_{j}=D^{i} W_{i j}-(1+\sigma) D^{i} W_{j i}+\sigma h^{k l} D_{j} W_{k l}, \\
& \partial_{t} W_{i j}=D_{i} E_{j}+\frac{\tau}{2} h_{i j} D^{k} E_{k}+D_{i} D_{j} \phi,
\end{aligned}
$$

subject to the constraints $C \equiv D^{i} E_{i}=0$ and $C_{i j} \equiv W_{i j}-D_{i} A_{j}=0$. Here, $\phi$ denotes the electrostatic potential, $h_{i j}$ the components of the Euclidean metric and $\sigma$ and $\tau$ are nonvanishing constants having the same sign.

A well-posed IBVP for this system is obtained in the following way: first, the constraint propagation system describing the time evolution of the constraint fields $C$ and $C_{i j}$ is analysed. It is given by

$$
\begin{aligned}
& \partial_{t} C_{i j}=\frac{\tau}{2} h_{i j} C, \\
& \partial_{t} C=-\sigma D^{i} C_{i}, \\
& \partial_{t} C_{i}=-\tau D_{i} C,
\end{aligned}
$$

where $C_{i} \equiv D^{j} C_{i j}-h^{k l} D_{i} C_{k l}$. By imposing, for instance, the boundary condition $N^{i} C_{i} \hat{=} 0$ one obtains an $L^{2}$ estimate for the constraint fields. Next, using this, the physical energy of the system and the radiative-type boundary condition

$$
\gamma_{i}^{j} E_{j}-N^{j}\left(W_{i j}-W_{j i}\right) \hat{=} g_{i},
$$

where $g_{i}$ is a given vector field on the boundary satisfying $N^{i} g_{i} \hat{=} 0$, one obtains $L^{2}$ bounds for the electric and magnetic fields, $E_{i}$ and $W_{[i j]}$, respectively. The remaining difficulty is to estimate the symmetric, gauge-dependent, part of $W_{i j}$. It turns out that the simplest gauge choice, the temporal gauge $\phi=0$, does not lead to such an estimate. This can be seen by means of the following family of electrostatic solutions [21]:

$$
A_{j}=t D_{j} f, \quad E_{j}=D_{j} f, \quad W_{i j}=t D_{i} D_{j} f,
$$

where $f$ is a smooth, time-independent, harmonic function. This family solves the evolution and constraint equations and the radiative-type boundary condition with boundary data $g_{i} \hat{=} \gamma_{i}{ }^{j} D_{j} f$. A problem is the time dependence of the magnetic potential which implies that for $t>0$ the solution depends on second derivatives of $f$ while the initial and boundary data depend only on first derivatives of $f$. This violates the expected energy estimate in $L^{2}$.

For this reason, in [21], a different gauge condition was introduced which can be obtained by minimizing the functional

$I_{E M}[\phi]=\frac{1}{2} \int_{\Sigma} h^{i j}\left(\partial_{t} A_{i}\right)\left(\partial_{t} A_{j}\right) \sqrt{h} \mathrm{~d}^{3} x=\frac{1}{2} \int_{\Sigma} h^{i j}\left(E_{i}+D_{i} \phi\right)\left(E_{j}+D_{j} \phi\right) \sqrt{h} \mathrm{~d}^{3} x$

over the space $H^{1}(\Sigma)$ consisting of square-integrable functions $\phi$ on $\Sigma$ which have squareintegrable first-order spatial derivatives. Therefore, this gauge condition minimizes the $L^{2}$ norm of the time deformation of the magnetic potential over all possible electrostatic potentials $\phi$ for which this norm is defined. Here and in the following, $\sqrt{h}$ and $h^{i j}$ denote, respectively, 
the determinant and the inverse of the 3-metric. In the above example, where $E_{j}=D_{j} f$, the minimum of $I_{E M}$ is zero and yields $\phi=-f$ which in turn implies that $A_{j}$ is time independent. Therefore, this gauge condition precludes the above counterexample. Furthermore, it was shown in [21] to yield a well-posed IBVP. A straightforward generalization of $I_{E M}$ is the family of functionals

$$
I_{E M, \mu}[\phi]=\frac{1}{2} \int_{\Sigma}\left[\mu^{2} \phi^{2}+h^{i j}\left(\partial_{t} A_{i}\right)\left(\partial_{t} A_{j}\right)\right] \sqrt{h} \mathrm{~d}^{3} x
$$

parametrized by a nonnegative constant $\mu$. The extra term, $\mu^{2} \phi^{2}$, in the integrand plays the role of a penalty term which prevents $|\phi|$ from becoming 'too large' and might be useful for numerical simulations. Variation of $I_{E M, \mu}$ with respect to $\phi$ yields the elliptic boundary value problem

$$
\begin{array}{ll}
\left(-\mu^{2}+D^{i} D_{i}\right) \phi+D^{i} E_{i}=0 & \text { on } \quad \Sigma, \\
N^{i}\left(D_{i} \phi+E_{i}\right) \hat{=} 0 & \text { on } \quad \partial \Sigma .
\end{array}
$$

Note that for $\mu>0$ the solution of this boundary value problem is unique while for $\mu=0$ the solution $\phi$ is only unique up to an additive constant which might cause problems in an elliptic solver. The important point here is the boundary condition (10) which allows us to set the normal component of the magnetic potential to zero at the boundary: $N^{j} A_{j} \hat{=} 0$. This boundary condition in turn allows us to estimate the $L^{2}$ norm of $\mathbf{A}$ and its first-order spatial derivatives in terms of the divergence and the curl of $\mathbf{A}$. The divergence of $\mathbf{A}$ can be estimated by taking the divergence of equation (1) and using equation (9). The curl of $\mathbf{A}$ is known since it corresponds to the magnetic field $W_{[i j]}$ for which an estimate has been obtained.

Using the methods of [21] one can show that the IBVP described by the evolution equations (1), (2), (3) for $\sigma \tau>0$, the boundary conditions (7) and $N^{i} C_{i} \hat{=} 0$ and the gauge condition (9), (10) is well-posed. The interesting point is that one still obtains a well-posed problem in the limiting case $\sigma=\tau=0$ where the evolution equations are weakly hyperbolic [23]. In this case, the constraints propagate tangentially to the boundary, and the boundary condition $N^{i} C_{i} \hat{=} 0$ has to be dropped.

\subsection{Einstein's field equations}

Next, we discuss how the ideas above can be applied to the IBVP of general relativity. In the remaining part of this section, we consider the full nonlinear Einstein equations with matter fields and draw particular attention to the propagation of the constraint fields, the propagation of the Weyl tensor and the gauge conditions. An IBVP for the nonlinear case is mentioned at the end of this section. In the next two sections, we prove that this problem is well posed in the weak field regime.

We start with Einstein's field equations in the $3+1$ formulation of [22]. This is done for simplicity; as discussed in the conclusions, other $3+1$ formulations might serve as a starting point. Assume spacetime $(M, g)$ is globally hyperbolic, i.e. there exists a globally defined time function $t: M \rightarrow I$ such that $M$ is foliated by spacelike hypersurfaces $\Sigma_{\tau}=\{p \in M: t(p)=\tau\}$. Let $n_{a}=-\alpha \nabla_{a} t$ be the future-pointing unit normal to these slices (we choose the time orientation such that the lapse function, $\alpha$, is strictly positive). The 3-metric $h_{a b}$ and extrinsic curvature $k_{a b}$ (first and second fundamental forms) are defined by

$$
\begin{aligned}
& h_{a b}=g_{a b}+n_{a} n_{b}, \\
& k_{a b}=-\nabla_{a} n_{b}-n_{a} a_{b},
\end{aligned}
$$

respectively, where $a_{b}=n^{a} \nabla_{a} n_{b}=D_{b}(\log \alpha)$ is the acceleration field. The $3+1$ form of Einstein's equations yields the evolution equations 


$$
\begin{aligned}
& \mathcal{L}_{n} h_{a b}=-2 k_{a b}, \\
& \mathcal{L}_{n} k_{a b}=R_{a b}-\frac{1}{\alpha} D_{a} D_{b} \alpha+k k_{a b}-2 k_{a}{ }^{d} k_{b d}-\kappa s_{a b},
\end{aligned}
$$

and the constraints

$$
\begin{aligned}
& H \equiv \frac{1}{2}\left(h^{a b} R_{a b}+k^{2}-k^{a b} k_{a b}\right)-\kappa \rho=0, \\
& M_{a} \equiv D_{a} k-D^{b} k_{a b}+\kappa j_{a}=0,
\end{aligned}
$$

where here and in the following, $D_{a}$ and $R_{a b}$ denote, respectively, the covariant derivative and the Ricci tensor compatible with the 3-metric $h_{a b}, k=h^{a b} k_{a b}$ is the trace of the extrinsic curvature, $\kappa=8 \pi G$ where $G$ is the gravitational constant, and where in terms of the stressenergy tensor $T_{a b}$ the source terms $\rho, j_{a}$ and $s_{a b}$ are given by

$$
\rho=n^{a} n^{b} T_{a b}, \quad j_{a}=-h_{a}{ }^{c} n^{d} T_{c d}, \quad s_{a b}=\left(h_{a}{ }^{c} h_{b}{ }^{d}-\frac{1}{2} h_{a b} g^{c d}\right) T_{c d} .
$$

For consistency with the Bianchi identities, the stress-energy tensor must be divergence-free which translates into the conditions

$$
\begin{aligned}
& \mathcal{L}_{n} \rho+\frac{1}{\alpha^{2}} D^{a}\left(\alpha^{2} j_{a}\right)-2 k \rho-\left(k^{a b}-k h^{a b}\right) s_{a b}=0 \\
& \mathcal{L}_{n} j_{a}+\frac{1}{\alpha^{2}} D_{a}\left(\alpha^{2} \rho\right)-k j_{a}+\frac{1}{\alpha} D^{b}\left(\alpha s_{a b}-\alpha h_{a b} h^{c d} s_{c d}\right)=0
\end{aligned}
$$

A well-known property of the evolution system (13), (14) is that the Ricci operator, $h_{a b} \mapsto R_{a b}$, is not elliptic. In particular, this implies that simple algebraic gauge choices yield a weakly hyperbolic system for which there are no general well-posedness results and standard discretizations lead to unstable numerical schemes [24-26]. A possible remedy to this problem is to adopt different gauge conditions, like the full harmonic gauge [27], in which the evolution equations become manifestly hyperbolic.

\subsection{Estimates for the constraint fields}

Independent of what gauge is chosen, the evolution system (13), (14) has the property that it induces 'nice' evolution equations for the constraint fields: as a consequence of the (twice contracted) Bianchi identity, the evolution equations (13), (14) and the consistency conditions (17), (18) one finds that $H$ and $M_{a}$ propagate according to the simple symmetric hyperbolic system

$$
\begin{aligned}
& \mathcal{L}_{n} H=\frac{1}{\alpha^{2}} D^{a}\left(\alpha^{2} M_{a}\right)+2 k H \\
& \mathcal{L}_{n} M_{a}=\frac{1}{\alpha^{2}} D_{a}\left(\alpha^{2} H\right)+k M_{a}
\end{aligned}
$$

which closely resembles a wave equation written in a first-order form. By specifying suitable boundary conditions for this system, estimates for the constraint fields $H$ and $M_{a}$ can be obtained. In the following, we shall assume that the timelike boundary $\mathcal{T}$ is such that $n^{a} v_{a} \geqslant 0$ everywhere on $\mathcal{T}$, where $v_{a}$ denotes the outward unit normal ${ }^{3}$ to $\mathcal{T}$. As can be seen

3 Different boundary conditions have to be specified for the case where $n^{a} v_{a}<0$ everywhere on $\mathcal{T}$. We do not analyse this case here. 
by a simple energy argument (cf lemma 2 ), the boundary condition

$$
\nu^{a} M_{a} \hat{=} 0
$$

guarantees that $H=0$ and $M_{a}=0$ everywhere on $[0, T] \times \Sigma$ provided $H=0$ and $M_{a}=0$ on the initial surface $\{0\} \times \Sigma$. Furthermore, the boundary condition (21) yields an $L^{2}$ estimate for the constraint fields $H$ and $M_{a}$ provided $\alpha, k$ and $n^{a}$ are sufficiently regular ${ }^{4}$. As we will see shortly, it turns out that we need more regularity and also need an $L^{2}$ estimate for the first spatial derivatives of $H$ and $M_{a}$. It will be shown in the linearized case that this can be achieved using the boundary condition (21).

Before we proceed, we make two comments. First, we stress that the evolution equations derived by Arnowitt-Deser-Misner [28] differ from that discussed here by the addition of the Hamiltonian constraint to the evolution equations for $k_{a b}$. As a consequence, in the former case, the resulting constraint propagation system is only weakly hyperbolic which makes it much more difficult to control the constraint fields. (See [29] for the details on this important difference.) Second, we would like to mention that if $n^{a} v_{a} \hat{=} 0$ and in the absence of matter fields, the boundary condition (21) corresponds to one of the four Einstein boundary conditions $v^{a} G_{a b} \hat{=} 0$, where $G_{a b}$ denotes the Einstein tensor, proposed in [30]. (In this case, equation (21) is equivalent to $v^{a} n^{b} G_{a b} \hat{=} 0$.) However, the imposition of all four Einstein boundary conditions can lead to difficulties. For example, imposing the two conditions $v^{a} n^{b} G_{a b} \hat{=} 0$ and $v^{a} v^{b} G_{a b} \hat{=} 0$ overdetermines the constraint propagation system since by virtue of the evolution equations the second condition is equivalent to setting $H \hat{=} 0$. Therefore, if the initial data violate the constraints (as is the case in most numerical simulations), a solution to the IBVP might not even exist.

\subsection{Estimates for the Weyl curvature}

The next step is to obtain estimates for the Weyl curvature tensor $C_{a b c d}$. In terms of the electric and magnetic parts of the Weyl tensor, defined by

$$
\begin{aligned}
& E_{b d} \equiv C_{a b c d} n^{a} n^{c}, \\
& B_{b d} \equiv \frac{1}{2} n^{a} C_{a b e f} \epsilon_{d}^{e f},
\end{aligned}
$$

where $\epsilon_{b c d} \equiv n^{a} \epsilon_{a b c d}$ denotes the natural volume element on $\left(\Sigma_{t}, h_{a b}\right)$, the Bianchi identities yield

$$
\begin{aligned}
& \mathcal{L}_{n} E_{a b}=-\frac{1}{\alpha^{2}} \epsilon_{c d(a} D^{c}\left(\alpha^{2} B^{d}{ }_{b)}\right)-5 k_{(a}{ }^{d} E_{b) d}+2 k E_{a b}+h_{a b} k^{c d} E_{c d}+P_{a b}, \\
& \mathcal{L}_{n} B_{a b}=+\frac{1}{\alpha^{2}} \epsilon_{c d(a} D^{c}\left(\alpha^{2} E^{d}{ }_{b)}\right)-5 k_{(a}{ }^{d} B_{b) d}+2 k B_{a b}+h_{a b} k^{c d} B_{c d}+Q_{a b},
\end{aligned}
$$

where

$$
\begin{aligned}
& P_{a b}=\frac{1}{2}\left[\frac{1}{\alpha^{2}} D_{(a}\left(\alpha^{2} M_{b)}\right)+2 k_{a b} H-\kappa\right. \\
& \left.\qquad\left(\mathcal{L}_{n} s_{a b}+k_{(a}{ }^{d} s_{b) d}+k_{a b} h^{c d} s_{c d}+\frac{1}{\alpha^{2}} D_{(a}\left(\alpha^{2} j_{b)}\right)-2 k_{a b} \rho\right)\right]^{T F}, \\
& Q_{a b}=-\frac{1}{2}\left[\epsilon_{c d(a}\left[k^{c}{ }_{b)} M^{d}+\kappa\left(D^{c} s^{d}{ }_{b)}-k^{c}{ }_{b)} j^{d}\right)\right]\right], \\
& { }^{4} \text { It is sufficient that they are measurable and almost everywhere bounded. }
\end{aligned}
$$


and the constraints on $E$ and $B$

$$
\begin{aligned}
& D^{b} E_{a b}+k^{c d} \epsilon_{d a}^{b} B_{c b}=P_{a}, \\
& D^{b} B_{a b}-k^{c d} \epsilon_{d a}^{b} E_{c b}=Q_{a},
\end{aligned}
$$

where

$$
\begin{aligned}
& P_{a}=\frac{1}{3} D_{a} H+\frac{1}{2}\left(k_{a}{ }^{b}-k h_{a}{ }^{b}\right) M_{b}+\kappa\left[\frac{1}{3} D_{a} \rho-\frac{1}{2}\left(k_{a}{ }^{b}-k h_{a}{ }^{b}\right) j_{b}-\frac{1}{2}\left(D^{b} s_{a b}-\frac{1}{3} h^{c d} D_{a} s_{c d}\right)\right] \\
& Q_{a}=-\frac{1}{2} \epsilon_{a}{ }^{c d}\left[D_{c} M_{d}+\kappa\left(k_{c}{ }^{b} s_{d b}-D_{c} j_{d}\right)\right] .
\end{aligned}
$$

In the above expressions for $P_{a}, Q_{a}, P_{a b}$ and $Q_{a b}$ we have used the constraint propagation equations (19), (20) in order to eliminate the Lie derivatives of the constraint fields $H$ and $M_{a}$ with respect to $n^{a}$.

Equations (24), (25) constitute a symmetric hyperbolic system for the fields $E$ and $B$ which bears a striking resemblance to Maxwell's equations. Note that the source terms $P_{a b}$ and $Q_{a b}$ depend on the constraint fields $H$ and $M_{a}$ and their first spatial derivatives as well as on first derivatives of the matter terms $s_{a b}$. This is the reason why more regularity is needed for the constraint fields (and the matter fields). A well-posed IBVP for the subsystem (24), (25) can be constructed using the method in [1] where suitable combinations of the constraint equations are added to the evolution equations (24), (25) (see also section 4.2). Boundary conditions for the resulting linear evolution system for $E$ and $B$ which lead to a well-posed problem ${ }^{5}$ in $L^{2}$ can then be specified in the following form [1]: set $N^{a}=h^{a i} N_{i}$ and choose for each $t \in[0, T]$ two unit vector fields $e_{2}^{a}$ and $e_{3}^{a}$ on $\{t\} \times \partial \Sigma$ which are mutually orthogonal, such that $N^{a}, e_{2}^{a}, e_{3}^{a}$ form an orthonormal set. Define $m^{a}=2^{-1 / 2}\left(e_{2}^{a}+\mathrm{i} e_{3}^{a}\right)$ and introduce the complex Newman-Penrose scalars

$$
\begin{aligned}
& \Psi_{0} \hat{=}\left[E_{a b}-\epsilon_{c d a} N^{c} B_{b}^{d}\right] m^{a} m^{b}, \\
& \Psi_{4} \hat{=}\left[E_{a b}+\epsilon_{c d a} N^{c} B_{b}^{d}\right] \bar{m}^{a} \bar{m}^{b} .
\end{aligned}
$$

Next, let $c$ be a smooth complex-valued function on $[0, T] \times \partial \Sigma$ with magnitude smaller than or equal to 1 , and let $q$ be a smooth complex-valued function on $[0, T] \times \partial \Sigma$. Boundary conditions can then be specified in the form $[1,13]$

$$
\Psi_{0} \hat{=} c \bar{\Psi}_{4}+q
$$

where an overbar denotes complex conjugation. The appearance of the complex conjugation in equation (34) can be understood as follows: under a rotation $m \mapsto \mathrm{e}^{\mathrm{i} \varphi} m$ of $m, \Psi_{0} \mapsto \mathrm{e}^{2 \mathrm{i} \varphi} \Psi_{0}$ while $\Psi_{4} \mapsto \mathrm{e}^{-2 \mathrm{i} \varphi} \Psi_{4}$. Therefore, if $q \mapsto \mathrm{e}^{2 \mathrm{i} \varphi} q$, the boundary condition (34) is invariant with respect to such rotations. Asymptotically, $\Psi_{0}$ and $\Psi_{4}$ represent, respectively, in- and outgoing gravitational radiation. Therefore, the function $c$ can be interpreted as a reflection coefficient.

\subsection{Estimates for the first and second fundamental forms}

So far, we have obtained estimates for the constraint fields and the curvature fields $E_{a b}$ and $B_{a b}$. It remains to estimate the 3-metric $h_{a b}$ and the extrinsic curvature $k_{a b}$. For this, we first note that the electric and magnetic components of the Weyl tensor can be

5 Here, we assume again that $n^{a}$ and the connection fields are measurable and almost everywhere bounded. 
expressed as

$$
\begin{aligned}
E_{a b} & =\frac{1}{2}\left[\mathcal{L}_{n} k_{a b}+R_{a b}+\frac{1}{\alpha} D_{a} D_{b} \alpha+k k_{a b}\right]^{T F}, \\
B_{a b} & =\epsilon_{c d(a} D^{c} k^{d}{ }_{b)},
\end{aligned}
$$

where $[\ldots]^{T F}$ denotes the trace-free part with respect to $h_{a b}$. This allows us to rewrite the evolution system (13), (14) as

$\mathcal{L}_{n} h_{a b}=-2 k_{a b}$,

$\mathcal{L}_{n} k_{a b}=E_{a b}-\frac{1}{\alpha} D_{a} D_{b} \alpha-k_{a}{ }^{c} k_{c b}+\frac{2}{3} h_{a b} H-\frac{\kappa}{2} s_{a b}+\frac{\kappa}{6} h_{a b}\left(4 \rho-h^{c d} s_{c d}\right)$.

Since estimates for $E_{a b}$ and $H$ have already been obtained, and provided suitable estimates for the matter terms $\rho$ and $s_{a b}$ are available, one should therefore be able to integrate this system and obtain estimates for $h_{a b}$ and $k_{a b}$. The question here is what precise estimates do we need. Since one would like to be able to estimate the Christoffel symbols, we need to control at least the $L^{2}$ norm of the 3-metric and its first-order spatial derivatives. Such an estimate can be obtained for fixed shift by integrating equation (37) provided a similar estimate for $k_{a b}$ is available. As in the electromagnetic case, one does not expect such an estimate to exist for any gauge condition for the lapse. Comparing equation (1) with equation (38) and making the analogy $A_{i} \leftrightarrow k_{a b}, E_{i} \leftrightarrow E_{a b}, \phi \leftrightarrow \alpha$ between the electromagnetic case and general relativity, the functional $I_{E M}$ suggests the consideration of the following functional over the space $H^{2}(\Sigma)$ consisting of square-integrable functions $\alpha$ on $\Sigma$ with square-integrable firstand second-order spatial derivatives,

$$
\begin{aligned}
I_{G R}[\alpha]= & \frac{1}{2} \int_{\Sigma} h^{a c} h^{b d}\left(\frac{\partial_{t} k_{a b}}{\alpha}\right)\left(\frac{\partial_{t} k_{c d}}{\alpha}\right) \sqrt{h} \mathrm{~d}^{3} x \\
= & \frac{1}{2} \int_{\Sigma} h^{a c} h^{b d}\left(\frac{1}{\alpha} D_{a} D_{b} \alpha-\frac{1}{\alpha} \mathcal{L}_{\beta} k_{a b}-F_{a b}\right) \\
& \times\left(\frac{1}{\alpha} D_{c} D_{d} \alpha-\frac{1}{\alpha} \mathcal{L}_{\beta} k_{c d}-F_{c d}\right) \sqrt{h} \mathrm{~d}^{3} x,
\end{aligned}
$$

where $F_{a b}=R_{a b}+k k_{a b}-2 k_{a}{ }^{d} k_{b d}-\kappa s_{a b}$ and $\beta^{a}$ is the shift vector field. The reason for introducing the factor $1 / \alpha$ in front of $\partial_{t} k_{a b}$ is that without this factor, the minimum of $I_{G R}$ is always zero and is attained for $\alpha=0$ when $\mathcal{L}_{\beta} k_{a b}$ vanishes ${ }^{6}$. Note that minima need not be unique: for example, if $\mathcal{L}_{\beta} k_{a b}=0$, the functional is invariant with respect to the rescaling $\alpha \mapsto \lambda \alpha$, where $\lambda$ is a nonvanishing constant. This rescaling corresponds to the coordinate reparametrization $t \mapsto \lambda^{-1} t$. Similarly to the electromagnetic case, the functional $I_{G R}$ can be generalized to

$$
I_{G R, \mu}[\alpha]=\frac{1}{2} \int_{\Sigma}\left[\mu^{2}\left(\alpha-\alpha_{0}\right)^{2}+h^{a c} h^{b d}\left(\frac{\partial_{t} k_{a b}}{\alpha}\right)\left(\frac{\partial_{t} k_{c d}}{\alpha}\right)\right] \sqrt{h} \mathrm{~d}^{3} x,
$$

where here, $\mu \geqslant 0$ and $\alpha_{0}$ is a given, strictly positive function on spacetime. The penalty term $\mu^{2}\left(\alpha-\alpha_{0}\right)^{2}$ might be useful in order to guarantee the uniqueness of minima and to ensure that the lapse is positive. Variation of $I_{G R, \mu}$ with respect to $\alpha$ yields the equation

$$
\mu^{2}\left(\alpha-\alpha_{0}\right)+\left(D^{a} D^{b}-\alpha \omega^{a b}-F^{a b}\right) \omega_{a b}=0
$$

6 This problem could be avoided by requiring an inhomogeneous boundary condition for the lapse; however, for our key estimate in lemma 1 it turns out that we need to vary $I_{G R}$ over the space of all $\alpha \in H^{2}(\Sigma)$. 
and the boundary conditions

$$
\begin{aligned}
& N^{a} N^{b} \omega_{a b} \hat{=} 0 \\
& N^{a} D^{b} \omega_{a b}+\mathcal{D}^{a}\left(\gamma_{a}^{b} \omega_{b c} N^{c}\right) \hat{=} 0
\end{aligned}
$$

where $\omega_{a b}=\left(D_{a} D_{b} \alpha-\mathcal{L}_{\beta} k_{a b}-\alpha F_{a b}\right) / \alpha^{2}$ and $\mathcal{D}$ denotes the covariant derivative on $\partial \Sigma$ induced by $D$. Below, we analyse the well posedness of the variational principle associated with $I_{G R, \mu}$ in the weak field limit and show that the resulting condition for the lapse leads to an $L^{2}$ estimate for $k_{a b}$ and its first-order spatial derivatives (see lemma 1).

A similar functional was recently used in [31] in order to construct a new geometric invariant measuring the amount of radiation contained in a data set and was also considered in [32]. Functionals very similar to $I_{G R}$ also arise in the theory of thin elastic plates (see, for instance, [33]).

\subsection{The IBVP for Einstein's vacuum field equations}

With the above considerations we may formulate the following IBVP for the Einstein vacuum field equations. The generalization to matter fields depends on the precise model for the matter. For this reason, from now on, we set $T_{a b}=0$.

1. Specify initial data $\left(h_{i j}, k_{i j}\right)$ on a three-dimensional Riemannian compact manifold with smooth boundary $\partial \Sigma$ satisfying the Hamiltonian and momentum constraint equations (15), (16).

2. Specify boundary data $q(t)$ on $[0, T] \times \partial \Sigma$. The initial and boundary data must satisfy certain compatibility conditions on the intersection $\{0\} \times \partial \Sigma$ of the initial surface with the timelike boundary (see, for example, $[3,4]$ ).

3. Choose the shift as an a priori specified vector field $\beta^{i}$ on $[0, T] \times \Sigma$ such that $\beta^{i} N_{i} \leqslant 0$ everywhere on $\mathcal{T}$. Note that this inequality is independent of the (yet unknown) metric.

4. Find a curve $t \mapsto\left(h_{i j}, k_{i j}\right)(t), 0 \leqslant t \leqslant T$, solution of the evolution equations (13), (14) with lapse satisfying the boundary value problem (39), (40), (41), such that

$$
\left(h_{i j}, k_{i j}\right)(0)=\left(h_{i j}, k_{i j}\right),
$$

and such that the boundary conditions

$$
N^{i} M_{i}(t) \hat{=} 0, \quad \Psi_{0}(t) \hat{=} c \bar{\Psi}_{4}(t)+q(t),
$$

hold, where $M_{i}=D_{i} k-D^{j} k_{i j}$ and $\Psi_{0}=\left[E_{i j}-\varepsilon_{k l i} N^{k} B_{j}^{l}\right] m^{i} m^{j}, \bar{\Psi}_{4}=\left[E_{i j}+\right.$ $\left.\varepsilon_{k l i} N^{k} B_{j}^{l}\right] m^{i} m^{j}$ with

$$
E_{i j}=\left[R_{i j}+k k_{i j}-k_{i}^{l} k_{l j}\right]^{T F}, \quad B_{i j}=\epsilon_{k l(i} D^{k} k_{j)}^{l} .
$$

In the following, we analyse the well posedness of this problem under simplifying assumptions. First, we only consider the weak field regime in which all the equations are linearized about the flat spacetime solution $\left(h_{i j}, k_{i j}\right)=\left(\delta_{i j}, 0\right), \alpha=1, \beta^{i}=0$. Second, we assume that the domain $\Sigma=(0,1) \times T^{2}$ is a cube where the two opposite faces $y=$ const and $z=$ const are identified with each other (although most of the results below are valid for more general domains). The main result of this paper is the well posedness of the above stated IBVP under these simplifying assumptions, see theorem 3. Results for more general cases will be discussed elsewhere [23]. 


\section{Gauge condition for the lapse}

In this section we analyse the well posedness of the variational principle for $I_{G R, \mu}$. As indicated above, for simplicity, we only consider here the case of linearization about flat spacetime. In this case, it is natural to set $\alpha_{0}=1$, and we expand $\alpha=1+f$ and ignore all terms in $I_{G R, \mu}$ which are cubic or higher order in $f$ and the perturbed metric and extrinsic curvature. This leads to the functional $I_{\mu}$ defined in equation (44) below where now $F_{i j}$ is equal to the linearized Ricci tensor.

Before we proceed, we fix some notation: we denote by $L^{2}(\Sigma, \mathbb{R}), L^{2}(\Sigma, V)$ and $L^{2}(\Sigma, S)$ the spaces of square-integrable functions, vector fields and symmetric tensor fields, respectively, on $\Sigma$. Similarly, $H^{m}(\Sigma, \mathbb{R}), H^{m}(\Sigma, V), H^{m}(\Sigma, S)$ denote the Sobolev spaces of order $m$ of smooth functions, vector fields and symmetric tensor fields on $\Sigma$ and $C^{\infty}(\bar{\Sigma}, \mathbb{R}), C^{\infty}(\bar{\Sigma}, V), C^{\infty}(\bar{\Sigma}, S)$ the space of functions, vector fields and symmetric tensor fields on $\bar{\Sigma}$ which are infinitely differentiable on $\Sigma$ and such that all derivatives have a continuous extension on $\bar{\Sigma}$.

Define the bounded linear operator

$$
W: H^{2}(\Sigma, \mathbb{R}) \rightarrow L^{2}(\Sigma, S), \quad f \mapsto W_{i j} f=D_{i} D_{j} f .
$$

We consider for each $\mu \geqslant 0$ and $\mathbf{F} \in L^{2}(\Sigma, S)$ the functional

$$
I_{\mu}: H^{2}(\Sigma, \mathbb{R}) \rightarrow \mathbb{R}, \quad f \mapsto \frac{1}{2} \int_{\Sigma}\left[\mu^{2} f^{2}+\left(W^{i j} f-F^{i j}\right)\left(W_{i j} f-F_{i j}\right)\right] \sqrt{h} \mathrm{~d}^{3} x .
$$

Theorem 1 (minimum of $I_{\mu}$ ). The functional $I_{\mu}: H^{2}(\Sigma, \mathbb{R}) \rightarrow \mathbb{R}$ has the following properties:

(i) There exists a global minimum $f \in H^{2}(\Sigma, \mathbb{R})$ of $I_{\mu}$. For $\mu>0$ this minimum is unique; for $\mu=0$ the minimum is unique up to the addition of an element in the two-dimensional set

$$
\mathcal{N}=\left\{f=a_{0}+a_{1} x: a_{0}, a_{1} \in \mathbb{R}\right\} .
$$

(ii) A global minimum $f \in H^{2}(\Sigma, \mathbb{R})$ of $I_{\mu}$ satisfies

$$
I_{\mu}[f] \leqslant \frac{1}{2}\|\mathbf{F}\|_{L^{2}(\Sigma, S)}^{2} .
$$

(iii) If $\mu=0$, a global minimum $f \in H^{2}(\Sigma$, $\mathbb{R})$ satisfies $(\mathbf{F}-\mathbf{W} f, \mathbf{W} f)_{L^{2}(\Sigma, S)}=0$, i.e. $\mathbf{F}-\mathbf{W} f$ and $\mathbf{W} f$ are orthogonal.

(iv) If $\mathbf{F} \in C^{\infty}(\bar{\Sigma}, S)$, all global minima $f \in H^{2}(\Sigma, \mathbb{R})$ automatically lie in $C^{\infty}(\bar{\Sigma}, S)$ and satisfy the elliptic boundary value problem

$$
\begin{aligned}
& \mu^{2} f+W^{i j}\left(W_{i j} f-F_{i j}\right)=0 \quad \text { on } \Sigma \text {, } \\
& N^{i} N^{j}\left(W_{i j} f-F_{i j}\right) \hat{=} 0 \quad \text { on } \partial \Sigma \text {, } \\
& N^{i}\left(D^{j}+\mathcal{D}^{j}\right)\left(W_{i j} f-F_{i j}\right) \hat{=} 0 \quad \text { on } \quad \partial \Sigma \text {, }
\end{aligned}
$$

where $\mathcal{D}$ denotes the covariant derivative on $\partial \Sigma$ induced by $D$.

Proof. The proof is based on standard arguments from elliptic theory. In a first step, we set $X \equiv H^{2}(\Sigma, \mathbb{R})$ and rewrite the functional $I_{\mu}$ in the form

$$
I_{\mu}[f]=Q_{\mu}(f, f)-2 J(f)+\frac{1}{2}\|\mathbf{F}\|_{L^{2}(\Sigma, S)}^{2},
$$


for $f \in X$, where the bounded bilinear form $Q_{\mu}: X \times X \rightarrow \mathbb{R}$ and the linear functional $J: X \rightarrow \mathbb{R}$ are defined by

$$
\begin{aligned}
& Q_{\mu}(f, g)=\frac{1}{2}\left[\mu^{2}(f, g)_{L^{2}(\Sigma, \mathbb{R})}+(\mathbf{W} f, \mathbf{W} g)_{L^{2}(\Sigma, S)}\right], \\
& J(g)=\frac{1}{2}(\mathbf{F}, \mathbf{W} g)_{L^{2}(\Sigma, S)},
\end{aligned}
$$

for $f, g \in X$. Since for all $f, g \in X$ and all $\varepsilon>0$ we have

$$
\frac{I_{\mu}[f+\varepsilon g]-I_{\mu}[f]}{\varepsilon}=2\left(Q_{\mu}(f, g)-J(g)\right)+\varepsilon Q_{\mu}(g, g)
$$

and since $Q_{\mu}(g, g) \geqslant 0$, it follows that $f \in X$ is a global minimum of $I_{\mu}$ if and only if

$$
Q_{\mu}(f, g)=J(g) \quad \text { for all } g \in X .
$$

(i) Therefore, we have to show that there exists $f \in X$ such that equation (47) holds. This is done using the theorem in appendix A which summarizes standard results from Fredholm theory. In order to apply the theorem, we first note that

$$
Q_{\mu}(f, f)+\frac{1}{2}\|f\|_{H^{1}(\Sigma, \mathbb{R})}^{2} \geqslant \frac{1}{2}\|f\|_{H^{2}(\Sigma, \mathbb{R})}^{2}
$$

for all $f \in X$. Next, set $Z=H^{1}(\Sigma, \mathbb{R})$ and denote by $\iota: X \rightarrow Z$ the inclusion which is compact since $\Sigma$ is bounded. Furthermore, we introduce the following linear bounded operators:

$$
\begin{aligned}
M: X \rightarrow X^{*}, & f \mapsto Q_{\mu}(f, .), \\
L: X \rightarrow X^{*}, & f \mapsto Q_{\mu}(f, .)+\frac{1}{2}(f, .)_{Z}, \\
R: Z \rightarrow X^{*}, & f \mapsto-\frac{1}{2}(f, .)_{Z} .
\end{aligned}
$$

With this notation, $f \in X$ satisfies equation (47) if and only if $M f=J$, and $M=L+K$ is the sum of the coercive operator $L$ and the compact operator $K=R \circ \iota$. According to theorem 4, a solution to this equation exists if and only if $J \in\left(\operatorname{ker} M^{t}\right)^{\perp}$, where $\left(M^{t} f\right)(g)=(M g)(f)$ for all $f, g \in X$ and $\left(\operatorname{ker} M^{t}\right)^{\perp}$ denotes the annihilator of $\operatorname{ker} M^{t}$. In our case, since $Q_{\mu}$ is symmetric,

$$
\left(M^{t} f\right)(g)=Q_{\mu}(g, f)=Q_{\mu}(f, g)=(M f)(g)
$$

for all $f, g \in X$; hence $M^{t}=M$. If $\mu>0$ we have $\operatorname{ker} M=\{0\}$ and so $\left(\operatorname{ker} M^{t}\right)^{\perp}=X^{*}$. Therefore, if $\mu>0$, there exists a unique global minimum $f \in X$. On the other hand, if $\mu=0$,

$$
\operatorname{ker} M=\left\{f \in X: W_{i j} f=0\right\}=\left\{a_{0}+a_{1} x: a_{0}, a_{1} \in \mathbb{R}\right\} .
$$

Furthermore, by definition, $J \in(\operatorname{ker} M)^{\perp}$. Hence, the existence of global minima follows from theorem 4.

(ii) Setting $\varepsilon=1, g=-f$ in equation (46) we obtain

$$
I_{\mu}[f]=I_{\mu}[0]-Q_{\mu}(f, f) \leqslant I_{\mu}[0]=\frac{1}{2}\|\mathbf{F}\|_{L^{2}(\Sigma, S)}^{2} .
$$

(iii) This follows from equation (47) by setting $g=f$.

(iv) This can be seen by explicitly computing the solution and using integration by parts.

Theorem 1 has the following physical interpretation: consider the linearized extrinsic curvature $k_{i j}$ and perform an infinitesimal coordinate transformation $\delta x^{a} \mapsto \delta x^{a}+X^{a}$ parametrized by the vector field $\left(X^{a}\right)=\left(X^{t}, X^{i}\right)$. With respect to this, the linearized extrinsic 
curvature transforms according to

$$
k_{i j} \mapsto k_{i j}-W_{i j} f,
$$

where $f=X^{t}$. Therefore, given $\mathbf{k} \in L^{2}(\Sigma, S)$, the minimization principle described by $I_{\mu}$ provides a unique way of gauge-fixing $k_{i j}^{(G F)} \equiv k_{i j}-W_{i j} f$ by choosing $f$ such that it minimizes

$$
I_{\mu}[f]=\frac{1}{2} \int_{\Sigma}\left[\mu^{2} f^{2}+h^{i k} h^{j l}\left(k_{i j}^{(G F)}\right)\left(k_{k l}^{(G F)}\right)\right] \sqrt{h} \mathrm{~d}^{3} x
$$

According to theorem 1(ii), this defines for each $\mu \geqslant 0$ a bounded linear map $P_{\mu}^{(G F)}$ : $L^{2}(\Sigma, S) \rightarrow L^{2}(\Sigma, S), \mathbf{k} \mapsto \mathbf{k}^{(G F)}$ whose operator norm is less than or equal to 1 . In particular, we have a unique decomposition

$$
k_{i j}=k_{i j}^{(G F)}+W_{i j} f
$$

for each $\mathbf{k} \in L^{2}(\Sigma, S)$, where $\mathbf{k}^{(G F)} \equiv P_{\mu}^{(G F)} \mathbf{k}$. For $\mu=0$ this decomposition is orthogonal and $P_{0}^{(G F)}$ is an orthogonal projector, i.e. $P_{0}^{(G F)} \circ P_{0}^{(G F)}=P_{0}^{(G F)}$ and $\left(P_{0}^{(G F)}\right)^{*}=P_{0}^{(G F)}$. If $\mathbf{k} \in C^{\infty}(\bar{\Sigma}, S)$, the same holds for $\mathbf{k}^{(G F)}$ and it satisfies

$$
\begin{array}{ll}
\mu^{2} f-W^{i j} k_{i j}^{(G F)}=0 & \text { on } \quad \Sigma, \\
N^{i} N^{j} k_{i j}^{(G F)} \hat{=} 0 & \text { on } \quad \partial \Sigma, \\
N^{i}\left(D^{j}+\mathcal{D}^{j}\right) k_{i j}^{(G F)} \hat{=} 0 & \text { on } \quad \partial \Sigma .
\end{array}
$$

One of the key properties of the gauge-fixing operator $P_{\mu}^{(G F)}$ that will be crucial in our well posedness proof below is the following: defining the curl and momentum operators by

$$
\begin{array}{lll}
\text { curl: } & H^{1}(\Sigma, S) \rightarrow L^{2}(\Sigma, S) & k_{i j} \mapsto(\operatorname{curl} \mathbf{k})_{i j} \equiv \varepsilon_{k l(i} D^{k} k_{j}^{l}, \\
\mathcal{M}: & H^{1}(\Sigma, S) \rightarrow L^{2}(\Sigma, V) & k_{i j} \mapsto(\mathcal{M k})_{i} \equiv D_{i} k-D^{j} k_{i j},
\end{array}
$$

the gauge-fixed linearized extrinsic curvature $\mathbf{k}^{(G F)}=P_{\mu}^{(G F)} \mathbf{k}$ has the property that its $H^{1}$ norm is already controlled by its $L^{2}$ norm and the $L^{2} \operatorname{norm}^{7}$ of $\operatorname{curl~k}^{(G F)}$ and $\mathcal{M} \mathbf{k}^{(G F)}$ :

Lemma 1. Let $\mu \geqslant 0$. There exists a constant $C=C_{\mu}>0$ such that

$$
\|\mathbf{k}\|_{H^{1}(\Sigma, S)} \leqslant C\left[\|\mathbf{k}\|_{L^{2}(\Sigma, S)}+\|\mathbf{c u r l} \mathbf{k}\|_{L^{2}(\Sigma, S)}+\|\mathcal{M} \mathbf{k}\|_{L^{2}(\Sigma, V)}\right]
$$

for all $\mathbf{k} \in P_{\mu}^{(G F)} C^{\infty}(\bar{\Sigma}, S)$.

Proof. Set $\left(N^{i}\right)=(1,0,0)$ and denote by $A, B, C, D, E, F$ indices running over $y$ and $z$. Define the quantities

$$
\begin{aligned}
& Q \equiv N^{i} N^{j}(\text { curl k })_{i j}=\varepsilon^{A B} D_{A} k_{B x}, \\
& R \equiv N^{i}(\mathcal{M} \mathbf{k})_{i}=D_{x} k^{B}{ }_{B}-D^{B} k_{x B}, \\
& V_{A}^{(+)} \equiv \gamma_{A j}\left[-\frac{1}{2}(\mathcal{M} \mathbf{k})^{j}+N_{i} \varepsilon^{i j k}(\text { curl k })_{k l} N^{l}\right]=D_{x} k_{x A}-D_{A} k_{x x}, \\
& V_{A}^{(-)} \equiv \gamma_{A j}\left[-\frac{1}{2}(\mathcal{M} \mathbf{k})^{j}-N_{i} \varepsilon^{i j k}(\operatorname{curl} \mathbf{k})_{k l} N^{l}\right]=D^{B} k_{A B}-D_{A} k^{B}{ }_{B}, \\
& q_{A B} \equiv-\gamma_{A i} N_{j} \varepsilon^{i j k}(\text { curl k })_{k l} \gamma_{B}^{l}=\left[D_{x} k_{A B}-D_{A} k_{x B}\right]^{t f},
\end{aligned}
$$

7 Note that $\operatorname{curl}^{(G F)}=\operatorname{curl} \mathbf{k}$ and $\mathcal{M} \mathbf{k}^{(G F)}=\mathcal{M} \mathbf{k}$. 
where $\left[v_{A B}\right]^{t f} \equiv v_{A B}-\frac{1}{2} \delta_{A B} \delta^{C D} v_{C D}$ denotes the trace-free part of a 2-tensor $v_{A B}$. Using integration by parts, we first find

$$
\begin{aligned}
\int_{\Sigma}\left[Q^{2}+R^{2}\right. & \left.+\gamma^{A B}\left(V_{A}^{(+)}+D_{A} k\right)\left(V_{B}^{(+)}+D_{B} k\right)\right] \mathrm{d}^{3} x \\
= & \int_{\Sigma}\left[\left(D_{x} k^{B}{ }_{B}\right)^{2}+\left(D^{A} k^{B}{ }_{B}\right)\left(D_{A} k^{C}{ }_{C}\right)+\left(D^{x} k^{x B}\right)\left(D_{x} k_{x B}\right)\right. \\
& \left.+\left(D^{A} k^{x B}\right)\left(D_{A} k_{x B}\right)\right] \mathrm{d}^{3} x-2 \int_{T^{2}}\left[\left(k^{B}{ }_{B}\right)\left(D^{A} k_{x A}\right)\right]_{x=0}^{1} \mathrm{~d} y \mathrm{~d} z .
\end{aligned}
$$

On the other hand, using the fact that $D^{i} D^{j} k_{i j}=\mu^{2} f$, we find

$2 \int_{\Sigma}\left[\left(D^{i} k\right)(\mathcal{M k})_{i}-\mu^{2} k f\right] \mathrm{d}^{3} x=2 \int_{\Sigma}\left(D^{i} k\right)\left(D_{i} k\right) \mathrm{d}^{3} x-2 \int_{T^{2}}\left[k D^{j} k_{x j}\right]_{x=0}^{1} \mathrm{~d} y \mathrm{~d} z$.

Adding equations (54) and (55) together, and using the boundary conditions $k_{x x}=0, D^{j} k_{x j}+$ $D^{A} k_{x A}=0$ on $\partial \Sigma$ we obtain

$$
\begin{aligned}
\int_{\Sigma}\left[\left(D^{i} k^{B}{ }_{B}\right)\right. & \left.\left(D_{i} k_{C}^{C}\right)+\left(D^{i} k^{x B}\right)\left(D_{i} k_{x B}\right)+2\left(D^{i} k\right)\left(D_{i} k\right)\right] \mathrm{d}^{3} x \\
= & \int_{\Sigma}\left[Q^{2}+R^{2}+\gamma^{A B}\left(V_{A}^{(+)}+D_{A} k\right)\left(V_{B}^{(+)}+D_{B} k\right)+2\left(D^{i} k\right)(\mathcal{M} \mathbf{k})_{i}-2 \mu^{2} k f\right] \mathrm{d}^{3} x \\
\leqslant & \int_{\Sigma}\left[Q^{2}+R^{2}+\left(1+K_{1}\right) \gamma^{A B} V_{A}^{(+)} V_{B}^{(+)}+K_{2}(\mathcal{M} \mathbf{k})^{i}(\mathcal{M} \mathbf{k})_{i}\right] \mathrm{d}^{3} x \\
& +\left(\frac{1}{K_{1}}+\frac{1}{K_{2}}\right) \int_{\Sigma}\left(D^{i} k\right)\left(D_{i} k\right) \mathrm{d}^{3} x+K_{3}\|\mathbf{k}\|_{L^{2}(\Sigma, S)}^{2},
\end{aligned}
$$

where $K_{1}, K_{2}$ and $K_{3}$ are positive constants and where we have used theorem 1(ii) in the last step in order to estimate $\mu f$. By choosing $K_{1}$ and $K_{2}$ large enough such that $K_{1}^{-1}+K_{2}^{-1}<2$ it follows that we can bound the $H^{1}$ norm of the components $k_{x x}, k_{x A}, k_{A}^{A}$ in terms of the right-hand side of (53).

It remains to bound the $H^{1}$ norm of $\hat{k}_{A B}=k_{A B}-\frac{1}{2} \delta_{A B} k_{C}^{C}$. For this, we first note the identity

$$
\varepsilon_{A}{ }^{C} \hat{k}_{C B}=-\hat{k}_{A C} \varepsilon^{C}{ }_{B}
$$

which is valid for any symmetric, trace-less 2-tensor $\hat{k}_{A B}$. This implies

$$
2 D_{[A} \hat{k}_{B] C}=\varepsilon_{A B} \varepsilon^{E F} D_{E} \hat{k}_{F C}=-\varepsilon_{A B} D^{E} k_{E D} \varepsilon^{D}{ }_{C} .
$$

Using this, we find

$$
\begin{aligned}
\int_{\Sigma}\left(D^{i} \hat{k}^{B C}\right)\left(D_{i} \hat{k}_{B C}\right) \mathrm{d}^{3} x= & \int_{\Sigma}\left[\left(D^{x} \hat{k}^{B C}\right)\left(D_{x} \hat{k}_{B C}\right)+\left(D^{A} \hat{k}^{B C}\right)\left(D_{B} \hat{k}_{A C}\right)\right. \\
& \left.+2\left(D^{A} \hat{k}^{B C}\right)\left(D_{[A} \hat{k}_{B] C}\right)\right] \mathrm{d}^{3} x \\
= & \int_{\Sigma}\left[\left(D^{x} \hat{k}^{B C}\right)\left(D_{x} \hat{k}_{B C}\right)+2\left(D_{B} \hat{k}^{B C}\right)\left(D^{A} \hat{k}_{A C}\right)\right] \mathrm{d}^{3} x \\
= & \int_{\Sigma} \gamma^{A C} \gamma^{B D}\left[q_{A B}+\left(D_{A} k_{x B}\right)^{t f}\right]\left[q_{C D}+\left(D_{C} k_{x D}\right)^{t f}\right] \mathrm{d}^{3} x \\
& +\frac{1}{2} \int_{\Sigma} \gamma^{A C}\left[2 V_{A}^{(-)}+D_{A} k_{B}^{B}\right]\left[2 V_{C}^{(-)}+D_{C} k_{D}^{D}\right] \mathrm{d}^{3} x,
\end{aligned}
$$

where we have used integration by parts in the second step. This proves the statement of the lemma. 


\section{Well-posed IBVP for the linearized vacuum equations}

In this section, we present a well-posed initial-boundary value formulation for the linearized Einstein vacuum equations when linearized about a flat spacetime. For simplicity, we also assume that $\Sigma=(0,1) \times T^{2}$ although most of the results below hold for the general case where $\Sigma \subset \mathbb{R}^{3}$ is an open bounded domain with $C^{\infty}$ boundary $\partial \Sigma$. In the following, let

$$
\left(N^{j}\right)=(1,0,0), \quad\left(m^{j}\right)=\frac{1}{\sqrt{2}}(0,1, i),
$$

such that $N^{j}, m^{j}, \bar{m}^{j}$ form a complex triad adapted to the boundary. We assume that the linearized shift is exactly zero and fix the gauge for the linearized lapse by requiring that $P^{(G F)} \mathbf{k}=\mathbf{k}$ where $P^{(G F)} \equiv P_{0}^{(G F)}$ is the gauge-fixing projection operator introduced in the previous section. The linearized equations have the form

$$
\frac{\mathrm{d}}{\mathrm{d} t} u=A u,
$$

where $u=(\mathbf{h}, \mathbf{k})$ denotes the components of the linearized 3-metric and extrinsic curvature and

$$
A\left(\begin{array}{l}
\mathbf{h} \\
\mathbf{k}
\end{array}\right)=\left(\begin{array}{c}
-2 \mathbf{k} \\
P^{(G F)} \operatorname{Ric} \mathbf{h}
\end{array}\right)
$$

where Ric is the linearized Ricci operator. The idea is to represent $A$ on an appropriate Hilbert space $H$ such that $A: D_{H} \subset H \rightarrow H$ generates a strongly continuous semigroup ${ }^{8}$ $P(t)=\exp (t A)$ on $H$. The unique solution of the abstract Cauchy problem

$$
\begin{array}{ll}
\frac{\mathrm{d}}{\mathrm{d} t} u=A u, & t>0, \\
u(t=0)=u_{0}, & u_{0} \in D_{H},
\end{array}
$$

is then given by $u(t)=P(t) u_{0}, t \geqslant 0$. Furthermore, the semigroup properties imply the existence of constants $a \geqslant 1, b \in \mathbb{R}$ such that $\|u(t)\| \leqslant a \exp (b t)\left\|u_{0}\right\|$ for all $t \geqslant 0$ and all $u_{0} \in H$. In particular, the problem is well posed.

Therefore, our task is to find a Hilbert space $H$ and a domain $D_{H} \subset H$ such that $A: D_{H} \subset H \rightarrow H$ generates a strongly continuous semigroup. There are well-known sufficient and necessary conditions for this to be the case, see for instance [34] and [35] and references therein for generalizations to quasilinear operators. In our case, the Hilbert space $H$ is motivated from our general considerations in section 2 and we will first define $A$ on the space of smooth tensor fields $(\mathbf{h}, \mathbf{k})$ satisfying the boundary conditions (the space $D_{0}$ below). Then, it will be shown that $A$ is quasi-dissipative ${ }^{9}$ and that it can be extended (by taking its closure in $H$ ) in such a way that the extension generates a strongly continuous semigroup on $H$. The following theorem gives the general structure of our Hilbert space $H$.

Theorem 2 (linear constrained evolution systems with curvature map). Let $X, Y, Z$ be real Hilbert spaces, and let

$$
\begin{array}{ll}
A: D(A) \subset X \rightarrow X & (\text { main evolution vector field), } \\
\hat{A}: D(\hat{A}) \subset Y \rightarrow Y & (\text { constraint evolution vector field), } \\
B: D(B) \subset Z \rightarrow Z & (\text { curvature evolution vector field), }
\end{array}
$$

8 That is, $P$ is a strongly continuous map from $[0, \infty)$ to $\mathcal{L}(H)$ satisfying $P(0)=\operatorname{id}_{H}$ and the semigroup property $P(t+s)=P(t) \circ P(s)$ for all $t, s \geqslant 0$.

$9 A$ is called dissipative if $(u, A u) \leqslant 0$ for all $u \in D(A)$ and quasi-dissipative if there exists a constant $b \in \mathbb{R}$ such that $A-b$ is dissipative. 
be densely-defined linear operators on $X, Y$ and $Z$, respectively. Further, let $D_{0} \subset D(A)$ be a dense linear subspace of $X$ and

$$
\begin{array}{ll}
S_{0}: D \subset X \rightarrow Y & \text { (constraint map), } \\
T_{0}: D \subset X \rightarrow Z & \text { (curvature map) }
\end{array}
$$

be closable linear operators such that $D_{0} \subset D, A\left(D_{0}\right) \subset D, S_{0}\left(D_{0}\right) \subset D(\hat{A}), T_{0}\left(D_{0}\right) \subset$ $D(B)$ and such that the following intertwining relations hold,

$$
\begin{aligned}
& S_{0} A u=\hat{A} S_{0} u, \\
& T_{0} A u=B T_{0} u+L_{1} S_{0} u+L_{0} u
\end{aligned}
$$

for all $u \in D_{0}$ where $L_{1}: Y \rightarrow Z, L_{0}: X \rightarrow Z$ are bounded linear maps. Furthermore, assume the existence of constants $a_{0}, a_{1}, a_{2}, \hat{a}, b \in \mathbb{R}$ such that

$$
\begin{array}{lll}
(u, A u)_{X} \leqslant a_{0}\|u\|_{X}^{2}+a_{1}\left\|S_{0} u\right\|_{Y}^{2}+a_{2}\left\|T_{0} u\right\|_{Z}^{2} & \text { for all } u \in D_{0}, \\
(c, \hat{A} c)_{Y} \leqslant \hat{a}\|c\|_{Y}^{2} & \text { for all } c \in D(\hat{A}), \\
(w, B w)_{Z} \leqslant b\|w\|_{Z}^{2} & \text { for all } w \in D(B) .
\end{array}
$$

Then,

(a) The operator $R_{0}: D \subset X \rightarrow Y \times Z$ defined by $R_{0} u=\left(S_{0} u, T_{0} u\right)$ for all $u \in D$, is closable. The domain $D(R)$ of its closure $R=\overline{R_{0}}$ is contained in $D(S) \cap D(T)$ where $S=\overline{S_{0}}, T=\overline{T_{0}}$ and $R u=(S u, T u)$ for all $u \in D(R)$. The linear space $H=D(R)$, equipped with the scalar product

$$
\begin{aligned}
(u, v)_{H} & \equiv(u, v)_{X}+(R u, R v)_{Y \times Z} \\
& =(u, v)_{X}+(S u, S v)_{Y}+(T u, T v)_{Z},
\end{aligned}
$$

for $u, v \in H$ defines a Hilbert space. Furthermore, the restriction of the operators $S$ and $T$ to $H$ are bounded linear operators from $H$ to $Y$ and $Z$, respectively.

(b) If $D_{0} \subset H$ is dense in $H$, the operator $A_{H}: D\left(A_{H}\right) \subset H \rightarrow H$ defined by

$$
D\left(A_{H}\right)=D_{0}, \quad A_{H} u=A u, \quad u \in D_{0},
$$

is densely-defined and quasi-dissipative. In particular, $A_{H}$ is closable.

(c) In addition, assume that $\left(\lambda-A_{H}\right) D_{0}$ is dense in $H$ for some sufficiently large $\lambda>0$. Then, $\overline{A_{H}}$ generates a strongly continuous semigroup $P:[0, \infty) \rightarrow \mathcal{L}(H)$ on $H$.

(d) If in addition $\overline{\hat{A}}$ generates a strongly continuous semigroup $Q:[0, \infty) \rightarrow \mathcal{L}(Y)$ on $Y$, then

$$
S P(t)=\left.Q(t) S\right|_{H}
$$

for all $t \geqslant 0$. In particular, $\left.\operatorname{ker} S\right|_{H}$ is left-invariant under $P(t)$ for all $t \geqslant 0$.

Proof. (a) Let $u_{n}$ be a sequence in $D$ which converges to zero in $X$ such that $R_{0} u_{n}=$ $\left(S_{0} u_{n}, T_{0} u_{n}\right)$ converges to some $(c, w) \in Y \times Z$. Since $S_{0}$ and $T_{0}$ are closable, it follows that $c=0$ and $w=0$. Therefore, $R_{0}$ is closable. Next, let $u \in D(R)$. Then, by definition of the closure, there exists a sequence $u_{n}$ in $D$ which converges to $u$ in $X$ such that $R_{0} u_{n} \rightarrow R u$. Since $R_{0} u_{n}=\left(S_{0} u, T_{0} u_{n}\right)$, it follows that $u \in D(S) \cap D(T)$ and that $R u=(S u, T u)$. Next, it is clear that $H$ is linear and that $(., .)_{H}$ defines a scalar product on $H$. The completeness of $H$ is an immediate consequence of the closedness of $R$. Finally, the fact that $\left.S\right|_{H}$ and $\left.T\right|_{H}$ are bounded is clear. 
(b) According to the assumptions, $A\left(D_{0}\right) \subset H$, so $A_{H}$ is a well-defined, linear operator. Next, let $u \in D_{0}$. Using the intertwining relations (64), (65) and the estimates (66) we obtain

$$
\begin{aligned}
\left(u, A_{H} u\right)_{H}= & (u, A u)_{X}+\left(S_{0} u, S_{0} A u\right)_{Y}+\left(T_{0} u, T_{0} A u\right)_{Z} \\
= & (u, A u)_{X}+\left(S_{0} u, \hat{A} S_{0} u\right)_{Y}+\left(T_{0} u, B T_{0} u+L_{1} S_{0} u+L_{0} u\right)_{Z} \\
\leqslant & a_{0}\|u\|_{X}^{2}+\left(a_{1}+\hat{a}\right)\left\|S_{0} u\right\|_{Y}^{2}+\left(a_{2}+b\right)\left\|T_{0} u\right\|_{Z}^{2} \\
& +\left\|L_{1}\right\|\left\|T_{0} u\right\|_{Z}\left\|S_{0} u\right\|_{Y}+\left\|L_{0}\right\|\left\|T_{0} u\right\|_{Z}\|u\|_{X} \\
\leqslant & K\|u\|_{H}^{2}
\end{aligned}
$$

for a sufficiently large constant $K>0$ which is independent of $u$. Since $A_{H}$ is densely-defined and quasi-dissipative it is closable ${ }^{10}$.

(c) This is a direct consequence of the Lumer-Phillips theorem ${ }^{11}$.

(d) For this, we first note that $\hat{A}$ is closable since according to our assumptions it is densely-defined and quasi-dissipative. In the next step we show that

$$
S \bar{A}_{H} u=\overline{\hat{A}} S u
$$

for all $u \in D\left(\overline{A_{H}}\right)$. Let $u \in D\left(\overline{A_{H}}\right)$. By definition of the closure, there exists a sequence $u_{n}$ in $D\left(A_{H}\right)=D_{0}$ such that $u_{n} \rightarrow u$ in $H$ and $A_{H} u_{n} \rightarrow \overline{A_{H}} u$ in $H$. Since for all $n \in \mathbb{N}, S A_{H} u_{n}=\hat{A} S u_{n}$ and since $\left.S\right|_{H}: H \rightarrow Y$ is bounded so that $S u_{n} \rightarrow S u$ in $Y$ and $S A_{H} u_{n} \rightarrow S \overline{A_{H}} u$ in $Y$, it follows that $S u \in D(\overline{\hat{A}})$ and that $\overline{\hat{A}} S u=S \bar{A}_{H} u$.

Next, let $u \in D\left(\overline{A_{H}}\right)$ and define the curve $c:[0, \infty) \rightarrow Y$ by $c(t)=S P(t) u, t \geqslant 0$. Since $\left.S\right|_{H}: H \rightarrow Y$ is linear and bounded, $c$ is differentiable on $(0, \infty)$ and

$$
\frac{\mathrm{d}}{\mathrm{d} t} c(t)=S \bar{A}_{H} P(t) u=\overline{\hat{A}} S P(t) u=\overline{\hat{A}} c(t)
$$

for all $t>0$. Since $c(0)=S u$ it follows by uniqueness of the Cauchy problem associated with $\overline{\hat{A}}$ that $c(t)=Q(t) S u$. Therefore, $S P(t) u=Q(t) S u$ for all $u \in D\left(\overline{A_{H}}\right)$. Since $D\left(\overline{A_{H}}\right)$ is dense in $H$ and $\left.S\right|_{H}: H \rightarrow Y$ is continuous the statement of the theorem follows.

In order to apply this theorem to the linearized vacuum equations, we define the operators $A, \hat{A}, B, S_{0}$ and $T_{0}$ and the function spaces $X, Y$ and $Z$ as follows. We start with the definition of the constraint evolution vector field $\hat{A}$.

\subsection{The constraint propagation system}

Define $Y=H^{1}(\Sigma, \mathbb{R}) \times\left\{\mathbf{M} \in H^{1}(\Sigma, V): N^{i} M_{i} \hat{=} 0\right\}$, and define the operator $\hat{A}: D(\hat{A}) \subset Y \rightarrow Y$ by

$$
\begin{aligned}
& D(\hat{A})=\left\{(H, \mathbf{M}) \in C^{\infty}(\bar{\Sigma}, \mathbb{R}) \times C^{\infty}(\bar{\Sigma}, V): N^{i} D_{i} H \hat{=} 0, N^{i} M_{i} \hat{=} 0\right\}, \\
& \hat{A}\left(\begin{array}{c}
H \\
\mathbf{M}
\end{array}\right)=\left(\begin{array}{c}
\operatorname{div} \mathbf{M} \\
\operatorname{grad} H
\end{array}\right),
\end{aligned}
$$

for all $(H, \mathbf{M}) \in D(\hat{A})$, where here and in the following we use the notation $\operatorname{div} \mathbf{M} \equiv D^{i} M_{i}$ and $(\operatorname{grad} H)_{i} \equiv D_{i} H$.

Lemma 2 (well posedness of the constraint propagation system). The operator $\hat{A}$ is a denselydefined, linear operator on $Y$ with the following properties:

${ }^{10}$ See theorem $4.5(\mathrm{c})$ in chapter 1 of [34] for a proof.

${ }^{11}$ See theorem 5.7 in [35] for a proof. 
(i) $\hat{A}$ is dissipative, that is, $(c, \hat{A} c)_{Y} \leqslant 0$ for all $c \in D(\hat{A})$.

(ii) For all $\lambda>0$

$$
(\lambda-\hat{A})(D(\hat{A}))=C^{\infty}(\bar{\Sigma}, \mathbb{R}) \times\left\{\mathbf{M} \in C^{\infty}(\bar{\Sigma}, V): N^{i} M_{i} \hat{=} 0\right\} .
$$

(iii) $\hat{A}$ is closable and its closure generates a strongly continuous semigroup $Q:[0, \infty) \rightarrow$ $\mathcal{L}(Y)$.

Proof. First, it is clear that $\hat{A}$ is linear and that $D(\hat{A})$ is dense in $Y$.

(i) Let $c=(H, \mathbf{M}) \in D(\hat{A})$. Using integration by parts we find

$$
\begin{aligned}
(c, \hat{A} c)_{Y} & =\int_{\Sigma}\left[H D^{i} M_{i}+\left(D^{j} H\right)\left(D_{j} D^{i} M_{i}\right)+M^{i} D_{i} H+\left(D^{j} M^{i}\right)\left(D_{j} D_{i} H\right)\right] \mathrm{d}^{3} x \\
& =\int_{\Sigma} D^{i}\left[H M_{i}+\left(D^{j} H\right)\left(D_{j} M_{i}\right)\right] \mathrm{d}^{3} x \\
& =\int_{T^{2}}\left[H M_{x}+\left(D^{j} H\right)\left(D_{j} M_{x}\right)\right]_{x=0}^{1} \mathrm{~d} y \mathrm{~d} z .
\end{aligned}
$$

Since $M_{x} \hat{=} D_{x} H \hat{=} 0$ the boundary integral vanishes and it follows that $\hat{A}$ is dissipative.

(ii) This follows using general theorems [3] about symmetric linear operators with maximal dissipative boundary conditions. However, in this particular case, it is not difficult to give a direct proof: let $\lambda>0$, and let $F \in C^{\infty}(\bar{\Sigma}, \mathbb{R})$ and $\mathbf{G} \in C^{\infty}(\bar{\Sigma}, V)$ with $N^{i} G_{i} \hat{=} 0$. We want to find $(H, \mathbf{M}) \in D(\hat{A})$ such that

$$
\begin{aligned}
& \lambda H-D^{i} M_{i}=F, \\
& \lambda M_{i}-D_{i} H=G_{i} .
\end{aligned}
$$

Equations (68), (69) and $(H, \mathbf{M}) \in D(\hat{A})$ imply the Neumann boundary-value problem

$$
\left(\lambda^{2}-D^{i} D_{i}\right) H=\lambda F+D^{i} G_{i}, \quad N^{i} D_{i} H \hat{=} 0,
$$

which has a unique solution $H \in C^{\infty}(\bar{\Sigma}, \mathbb{R})$. Setting $\mathbf{M}=\lambda^{-1}(\mathbf{G}+\operatorname{grad} H) \in C^{\infty}(\bar{\Sigma}, V)$ it follows that $(H, \mathbf{M}) \in D(\hat{A})$ satisfies $(\lambda-\hat{A})(H, \mathbf{M})=(F, \mathbf{G})$.

(iii) Since $C^{\infty}(\bar{\Sigma}, \mathbb{R}) \times\left\{\mathbf{M} \in C^{\infty}(\bar{\Sigma}, V): N^{i} M_{i} \hat{=} 0\right\}$ is dense in $Y$ this follows from (i) and (ii) and the Lumer-Phillips theorem ${ }^{12}$.

\subsection{The Weyl propagation system}

Next, we analyse the propagation of the Weyl curvature. As discussed in section 2 we describe the evolution of the Weyl tensor by the system constructed in [1]. For this, we define $Z=L^{2}(\Sigma, S) \times L^{2}(\Sigma, S)$, and let $B: D(B) \subset Z \rightarrow Z$ be the densely-defined linear operator

$$
\begin{aligned}
& D(B)=\left\{(\mathbf{E}, \mathbf{B}) \in C^{\infty}(\bar{\Sigma}, S) \times C^{\infty}(\bar{\Sigma}, S): \Psi_{0}[\mathbf{E}, \mathbf{B}] \hat{=} c \bar{\Psi}_{4}[\mathbf{E}, \mathbf{B}]\right\}, \\
& B\left(\begin{array}{l}
\mathbf{E} \\
\mathbf{B}
\end{array}\right)=\left(\begin{array}{c}
\left.-(\operatorname{curl} \mathbf{B})_{i j}+N_{(i} \varepsilon_{j}\right){ }^{k l} N_{k}(\operatorname{div} \mathbf{B})_{l} \\
(\operatorname{curl} \mathbf{E})_{i j}-N_{(i} \varepsilon_{j}{ }^{k l} N_{k}(\operatorname{div} \mathbf{E})_{l}
\end{array}\right),
\end{aligned}
$$

for all $(\mathbf{E}, \mathbf{B}) \in D(B)$. Here, $c$ is a complex constant of magnitude smaller than or equal to 1 and the maps $(\mathbf{E}, \mathbf{B}) \mapsto \Psi_{0}[\mathbf{E}, \mathbf{B}]$ and $(\mathbf{E}, \mathbf{B}) \mapsto \bar{\Psi}_{4}[\mathbf{E}, \mathbf{B}]$ are defined by

$$
\begin{aligned}
& \Psi_{0}[\mathbf{E}, \mathbf{B}]=\left[E_{i j}-\epsilon_{k l i} N^{k} B^{l}{ }_{j}\right] m^{i} m^{j}, \\
& \bar{\Psi}_{4}[\mathbf{E}, \mathbf{B}]=\left[E_{i j}+\epsilon_{k l i} N^{k} B^{l}{ }_{j}\right] m^{i} m^{j} .
\end{aligned}
$$

${ }^{12}$ See theorem 5.7 in [35] for a proof. 
As shown in [1], the operator $B$ is symmetric with maximally dissipative boundary conditions. The following lemma is a consequence of general theorems [3].

Lemma 3 (well posedness of the Weyl propagation system). The operator $B$ is a denselydefined, linear operator on $Z$ with the following properties:

(i) $B$ is dissipative.

(ii) For all $\lambda>0$

$$
(\lambda-B)(D(B))=C^{\infty}(\bar{\Sigma}, S) \times C^{\infty}(\bar{\Sigma}, S) .
$$

(iii) B is closable and its closure generates a strongly continuous semigroup on Z.

\subsection{The main evolution system}

Next, we turn our attention to the main evolution system. Using the gauge-fixing projection operator $P^{(G F)} \equiv P_{0}^{(G F)}$ introduced in the previous section we define the Hilbert space $X=H^{1}(\Sigma, S) \times P^{(G F)} L^{2}(\Sigma, S)$ and the dense subspace

$$
D=\left\{(\mathbf{h}, \mathbf{k}) \in C^{\infty}(\bar{\Sigma}, S) \times P^{(G F)} C^{\infty}(\bar{\Sigma}, S): N^{i}(\mathcal{M} \mathbf{k})_{i} \hat{=} 0\right\} .
$$

In terms of the linearized Ricci operator Ric $: H^{2}(\Sigma, S) \rightarrow L^{2}(\Sigma, S)$ defined by

$$
(\operatorname{Rich})_{i j}=D_{(i} D^{k} h_{j) k}-\frac{1}{2}\left(D^{k} D_{k} h_{i j}+\delta^{k l} D_{i} D_{j} h_{k l}\right), \quad \mathbf{h} \in H^{2}(\Sigma, S),
$$

the main evolution vector field is given by $A: D(A) \subset X \rightarrow X$ where $D(A)=D$ and

$$
A\left(\begin{array}{l}
\mathbf{h} \\
\mathbf{k}
\end{array}\right)=\left(\begin{array}{c}
-2 \mathbf{k} \\
P^{(G F)} \operatorname{Ric} \mathbf{h}
\end{array}\right)
$$

for all $(\mathbf{h}, \mathbf{k}) \in D$.

Next, we define the constraint map $S_{0}: D \subset X \rightarrow Y$ and the curvature map $T_{0}: D \subset X \rightarrow Z$ by

$$
S_{0}\left(\begin{array}{l}
\mathbf{h} \\
\mathbf{k}
\end{array}\right)=\left(\begin{array}{c}
\mathcal{H} \mathbf{h} \\
\mathcal{M} \mathbf{k}
\end{array}\right), \quad T_{0}\left(\begin{array}{l}
\mathbf{h} \\
\mathbf{k}
\end{array}\right)=\left(\begin{array}{c}
{\left[\operatorname{Ric} \mathbf{h}^{T F}\right.} \\
\mathbf{c u r l} \mathbf{k}
\end{array}\right), \quad\left(\begin{array}{l}
\mathbf{h} \\
\mathbf{k}
\end{array}\right) \in D
$$

where the linearized Hamiltonian operator $\mathcal{H}: H^{2}(\Sigma, S) \rightarrow L^{2}(\Sigma, \mathbb{R})$ is defined by

$$
\mathcal{H} \mathbf{h}=\frac{1}{2} \delta^{i j}(\operatorname{Ric} \mathbf{h})_{i j}=\frac{1}{2}\left(D^{i} D^{j} h_{i j}-\delta^{i j} D^{k} D_{k} h_{i j}\right), \quad \mathbf{h} \in H^{2}(\Sigma, S) .
$$

Finally, we define the subspace $D_{0}$ of $D$ as the subspace of $X$ consisting of smooth fields satisfying the boundary conditions. More precisely,

$$
\begin{aligned}
& D_{0}=\left\{(\mathbf{h}, \mathbf{k}) \in C^{\infty}(\bar{\Sigma}, S) \times P^{(G F)} C^{\infty}(\bar{\Sigma}, S):\right. \\
& N^{i}(\mathcal{M} \mathbf{k})_{i} \hat{=} 0 \\
& N^{i} D_{i}(\mathcal{H} \mathbf{h}) \hat{=} 0 \\
& \left.\Psi_{0}\left[T_{0}(\mathbf{h}, \mathbf{k})\right] \hat{=} c \bar{\Psi}_{4}\left[T_{0}(\mathbf{h}, \mathbf{k})\right]\right\},
\end{aligned}
$$

where $\Psi_{0}$ and $\bar{\Psi}_{4}$ are the maps defined in equations (70), (71) and $c$ is the complex constant appearing in the definition of $D(B)$. For the proof below, we require that $c \neq-1$ which excludes the case of a 'conducting boundary' described by $m^{i} m^{j} E_{i j} \hat{=} 0$.

In the next lemma and propositions 1 and 2 we show that the assumptions of theorem 2 are satisfied. This leads to the main result in theorem 3 . 


\section{Lemma 4.}

(i) The operators $S_{0}$ and $T_{0}$ are closable.

(ii) $A\left(D_{0}\right) \subset D, S_{0}\left(D_{0}\right) \subset D(\hat{A}), T_{0}\left(D_{0}\right) \subset D(B)$ and

$$
S_{0} A u=\hat{A} S_{0} u, \quad T_{0} A u=B T_{0} u+L_{1} S_{0} u,
$$

for all $u \in D_{0}$ where $L_{1}: Y \rightarrow Z$ is the bounded linear operator defined by

$$
L_{1}\left(\begin{array}{c}
H \\
\mathbf{M}
\end{array}\right)=\left(\begin{array}{c}
\frac{1}{2} D_{(i} M_{j)}-\frac{1}{6} \delta_{i j} D^{k} M_{k}+\frac{1}{2} N_{(i} \varepsilon_{j)}{ }^{k l} N_{k}(\mathbf{c u r l} \mathbf{M})_{l} \\
\frac{1}{3} N_{(i} \varepsilon_{j}{ }^{k l} N_{k} D_{l} H
\end{array}\right) .
$$

for all $(H, \mathbf{M}) \in Y$.

(iii) There are constants $a_{0}, a_{1}, a_{2} \in \mathbb{R}$ such that

$$
(u, A u)_{X} \leqslant a_{0}\|u\|_{X}^{2}+a_{1}\left\|S_{0} u\right\|_{Y}^{2}+a_{2}\left\|T_{0} u\right\|_{Z}^{2}
$$

for all $u \in D_{0}$.

Proof. See appendix B.

Remark 1. In the weak field limit of the IBVP considered here, the linear operator $L_{0}$ appearing in the intertwining relation (65) is identically zero. However, when considering the linearized Einstein equations about nonflat backgrounds, $L_{0} u$ involves the linearized connection fields and may be nonzero.

Following theorem 2 we denote by $R$ the closure of the operator $R_{0}: D \subset X \rightarrow Y \times Z$ defined by $R_{0} u=\left(S_{0} u, T_{0} u\right)$ for all $u \in D$, introduce the Hilbert space $H=D(R)$ equipped with the scalar product

$$
(u, v)_{H} \equiv(u, v)_{X}+(S u, S v)_{Y}+(T u, T v)_{Z}
$$

for $u, v \in H$ and define the linear operator $A_{H}: D_{0} \subset H \rightarrow H$ by $A_{H} u=A u$, for $u \in D_{0}$. It remains to prove the following two propositions:

Proposition 1. $D_{0} \subset H$ is dense.

Proposition 2. $(\lambda-A)\left(D_{0}\right)=D$ for all $\lambda>0$.

For the proof of proposition 1, we rely on the following two lemmas which are proven in appendix B.

Lemma 5. Given $\varepsilon>0$ and $G \in C^{\infty}(\partial \Sigma, \mathbb{R})$ there exists $\mathbf{h} \in C^{\infty}(\bar{\Sigma}, S)$ such that

$$
N^{i} D_{i}(\mathcal{H} \mathbf{h}) \hat{=} G
$$

and

$$
\|\mathbf{h}\|_{H^{1}(\Sigma, S)}<\varepsilon, \quad\|\operatorname{Ric} \mathbf{h}\|_{L^{2}(\Sigma, S)}<\varepsilon, \quad\|\mathcal{H} \mathbf{h}\|_{H^{1}(\Sigma, \mathbb{R})}<\varepsilon
$$

Lemma 6. Given $\varepsilon>0$ and $q \in C^{\infty}(\partial \Sigma, \mathbb{C})$ there exists $\mathbf{k} \in C^{\infty}(\bar{\Sigma}, S)$ such that

$$
N^{i}(\mathcal{M} \mathbf{k})_{i} \hat{=} 0, \quad(\text { curl } \mathbf{k})_{i j} m^{i} m^{j} \hat{=} q
$$

and

$$
\|\mathbf{k}\|_{L^{2}(\Sigma, S)}<\varepsilon, \quad\|\operatorname{curl} \mathbf{k}\|_{L^{2}(\Sigma, S)}<\varepsilon, \quad\|\mathcal{M} \mathbf{k}\|_{H^{1}(\Sigma, V)}<\varepsilon
$$


Proof of proposition 1. Let $u=(\mathbf{h}, \mathbf{k}) \in H$ and $\varepsilon>0$ be given. We have to show that there exists $\hat{u} \in D_{0}$ such that $\|\hat{u}-u\|_{H}<\varepsilon$. In order to do so, we first note that $D$ is dense in $H$ and pick $\bar{u}=(\overline{\mathbf{h}}, \overline{\mathbf{k}}) \in D$ such that

$$
\|\bar{u}-u\|_{H}^{2}<\frac{\varepsilon^{2}}{2} \text {. }
$$

Next, we use lemma 5 and choose $\tilde{\mathbf{h}} \in C^{\infty}(\bar{\Sigma}, S)$ such that $N^{i} D_{i}(\mathcal{H} \tilde{\mathbf{h}}) \hat{=}-N^{i} D_{i}(\mathcal{H} \overline{\mathbf{h}})$ and

$$
\|\tilde{\mathbf{h}}\|_{H^{1}(\Sigma, S)}^{2}<\frac{\varepsilon^{2}}{12}, \quad\|\operatorname{Ric} \tilde{\mathbf{h}}\|_{L^{2}(\Sigma, S)}^{2}<\frac{\varepsilon^{2}}{12}, \quad\|\mathcal{H} \tilde{\mathbf{h}}\|_{H^{1}(\Sigma, \mathbb{R})}^{2}<\frac{\varepsilon^{2}}{12} .
$$

Next, we note that the boundary condition $\Psi_{0}[\mathbf{E}, \mathbf{B}] \hat{=} c \bar{\Psi}_{4}[\mathbf{E}, \mathbf{B}]$ is equivalent to

$$
B_{i j} m^{i} m^{j} \hat{=} \frac{1}{i} \frac{1-c}{1+c} E_{i j} m^{i} m^{j}
$$

Therefore, we use lemma 6 and pick $\tilde{\mathbf{k}} \in C^{\infty}(\bar{\Sigma}, S)$ such that $\mathcal{M} \tilde{\mathbf{k}} \hat{=} 0$,

$$
\operatorname{curl}[\overline{\mathbf{k}}+\tilde{\mathbf{k}}]_{i j} m^{i} m^{j} \hat{=} \frac{1}{i} \frac{1-c}{1+c}[\operatorname{Ric}(\overline{\mathbf{h}}+\tilde{\mathbf{h}})]_{i j} m^{i} m^{j}
$$

and

$$
\|\tilde{\mathbf{k}}\|_{L^{2}(\Sigma, S)}^{2}<\frac{\varepsilon^{2}}{12}, \quad\|\operatorname{curl} \tilde{\mathbf{k}}\|_{L^{2}(\Sigma, S)}^{2}<\frac{\varepsilon^{2}}{12}, \quad\|\mathcal{M} \tilde{\mathbf{k}}\|_{H^{1}(\Sigma, V)}^{2}<\frac{\varepsilon^{2}}{12} .
$$

Finally, set $\hat{u}=\left(\overline{\mathbf{h}}+\tilde{\mathbf{h}}, \overline{\mathbf{k}}+P^{(G F)} \tilde{\mathbf{k}}\right)$. Noting that $\mathcal{M} P^{(G F)} \tilde{\mathbf{k}}=\mathcal{M} \tilde{\mathbf{k}}$ and $\operatorname{curl} P^{(G F)} \tilde{\mathbf{k}}=\mathbf{c u r l} \tilde{\mathbf{k}}$ it follows that $\hat{u} \in D_{0}$ and that

$$
\begin{aligned}
\|\hat{u}-u\|_{H}^{2} \leqslant & \|\hat{u}-\bar{u}\|_{H}^{2}+\|\bar{u}-u\|_{H}^{2} \\
= & \|\tilde{\mathbf{h}}\|_{H^{1}(\Sigma, S)}^{2}+\left\|P^{(G F)} \tilde{\mathbf{k}}\right\|_{L^{2}(\Sigma, S)}^{2}+\|\mathcal{H} \tilde{\mathbf{h}}\|_{H^{1}(\Sigma, \mathbb{R})}^{2}+\|\mathcal{M} \mathbf{k}\|_{H^{1}(\Sigma, V)}^{2} \\
& +\left\|[\operatorname{Ric} \tilde{\mathbf{h}}]^{T F}\right\|_{L^{2}(\Sigma, S)}^{2}+\|\operatorname{curl} \mathbf{k}\|_{L^{2}(\Sigma, S)}^{2}+\|\bar{u}-u\|_{H}^{2} \\
< & \varepsilon^{2}
\end{aligned}
$$

where we have used the fact that $P^{(G F)}$ is a projector in the last step.

Proof of proposition 2. Let $\lambda>0$ and $F \in D$. In the following, we construct $u=(\mathbf{h}, \mathbf{k}) \in D_{0}$ such that $(\lambda-A) u=F$. For this, we first use lemma 2(ii) and find $(H, \mathbf{M}) \in D(\hat{A})$ such that

$$
(\lambda-\hat{A})\left(\begin{array}{c}
H \\
\mathbf{M}
\end{array}\right)=S_{0} F .
$$

Next, using lemma 3(ii) we find $(\mathbf{E}, \mathbf{B}) \in D(B)$ such that

$$
(\lambda-B)\left(\begin{array}{l}
\mathbf{E} \\
\mathbf{B}
\end{array}\right)=T_{0} F+L_{1}\left(\begin{array}{l}
H \\
\mathbf{M}
\end{array}\right) .
$$

In the next step, we define the auxiliary variables

$$
\mathbf{Q}=\operatorname{div} \mathbf{E}-\frac{1}{3} \operatorname{grad} H, \quad \mathbf{P}=\operatorname{div} \mathbf{B}+\frac{1}{2} \operatorname{curl} \mathbf{M},
$$

and prove that they vanish. Multiplying $\mathbf{Q}$ and $\mathbf{P}$ by $\lambda$ and using equations (73), (74) we obtain the system

$$
\lambda\left(\begin{array}{c}
Q_{x} \\
Q_{A} \\
P_{x} \\
P_{A}
\end{array}\right)=\left(\begin{array}{c}
-\varepsilon^{A B} D_{A} P_{B} \\
-\frac{1}{2} \varepsilon_{A}^{B} D_{B} P_{x} \\
\varepsilon^{A B} D_{A} Q_{B} \\
\frac{1}{2} \varepsilon_{A}^{B} D_{B} Q_{x}
\end{array}\right)
$$


where $A, B$ run over $y$ and $z$. Multiplying both sides from the left with $\left(Q^{x}, 2 Q^{A}, P^{x}, 2 P^{A}\right)$ and integrating over $\Sigma$, it follows that $\mathbf{P}=\mathbf{Q}=0$, as claimed.

Next, we set

$$
u=\left(\begin{array}{l}
\mathbf{h} \\
\mathbf{k}
\end{array}\right)=\frac{1}{\lambda}\left[F+\left(\begin{array}{c}
-2 \mathbf{k} \\
P^{(G F)}\left(\mathbf{E}+\frac{2}{3} \delta H\right)
\end{array}\right)\right] .
$$

Since $N^{i} D_{i} H \hat{=} 0$ and $\mathbf{Q}=0$ it follows that $N^{i} \mathcal{M}\left(\mathbf{E}+\frac{2}{3} \delta H\right)_{i} \hat{=}-N^{i} \operatorname{div} \mathbf{E}_{i}+\frac{4}{3} N^{i} D_{i} H \hat{=} 0$. Therefore, it follows that $u \in D$. Next, using equation (73) and $\mathbf{Q}=0$ we find

$$
\lambda\left[\left(\begin{array}{l}
H \\
\mathbf{M}
\end{array}\right)-S_{0} u\right]=\left(\begin{array}{c}
\operatorname{div} \mathbf{M}-\operatorname{div} \mathcal{M} \mathbf{k} \\
0
\end{array}\right),
$$

which proves that $(H, \mathbf{M})=S_{0} u$. Finally, equation (74), $\mathbf{P}=\mathbf{Q}=0$ and $\mathbf{M}=\mathcal{M} \mathbf{k}$ yield

$$
\lambda\left[\left(\begin{array}{l}
\mathbf{E} \\
\mathbf{B}
\end{array}\right)-T_{0} u\right]=\left(\begin{array}{c}
-\operatorname{curl} \mathbf{B}+\operatorname{curl}^{2} \mathbf{k} \\
0
\end{array}\right),
$$

which shows that $(\mathbf{E}, \mathbf{B})=T_{0} u$. Therefore, $u \in D_{0}$ and $(\lambda-A) u=F$.

To summarize, we have shown:

Theorem 3. (main result) Let $c \in \mathbb{C}$ be such that $|c| \leqslant 1$ and $c \neq-1$. The operator $A: D_{0} \subset H \rightarrow H$ (cf equation (57)) describing the linearized Einstein evolution equations is closable and its closure generates a strongly continuous semigroup $P:[0, \infty) \rightarrow \mathcal{L}(H)$ on $H$. In particular, given initial data $u_{0}=(\mathbf{h}, \mathbf{k}) \in C^{\infty}(\bar{\Sigma}, S) \times C^{\infty}(\bar{\Sigma}, S)$ satisfying the gauge condition $P^{(G F)} \mathbf{k}=\mathbf{k}$ and the compatibility conditions

$$
\begin{aligned}
& N^{i}\left(D_{i} k-D^{j} k_{i j}\right) \hat{=} 0, \\
& N^{i} D_{i}\left(D^{k} D^{l} h_{k l}-\delta^{k l} D^{j} D_{j} h_{k l}\right) \hat{=} 0, \\
& \Psi_{0}\left[T_{0}(\mathbf{h}, \mathbf{k})\right] \hat{=} c \bar{\Psi}_{4}\left[T_{0}(\mathbf{h}, \mathbf{k})\right],
\end{aligned}
$$

where $\Psi_{0}$ and $\bar{\Psi}_{4}$ are the maps defined in equations (70), (71) and $T_{0}$ is the curvature map defined in equation (72), the curve $u:[0, \infty) \rightarrow H, t \mapsto u(t) \equiv P(t) u_{0}$ is continuous and differentiable on $(0, \infty)$ and is the unique solution of the abstract Cauchy problem (58). Furthermore, if $u_{0}$ satisfies the constraints, i.e. if $S_{0} u_{0}=0$, the constraints are satisfied for all $t>0$, i.e. $S u(t)=0$ for all $t>0$.

Remark 2. For each $t>0$ the solution $u(t)$ lies not only in $H$ but in the domain $D\left(\overline{A_{H}}\right)$ of the closure $\overline{A_{H}}$ of $A_{H}$. We do not intend to give an explicit representation of this space in this paper.

Remark 3. It should be possible to show that the solution is smooth provided extra compatibility conditions are required for the initial data.

Remark 4. Using Duhamel's principle ${ }^{13}$, it is not difficult to generalize the theorem to inhomogeneous boundary conditions and to the presence of source terms in the evolution equation.

${ }^{13}$ See, for example, corollary 2.11 in chapter 4 of [34]. 


\section{Conclusions}

In this paper we have discussed some new ideas for tackling the problem of obtaining a well-posed IBVP for metric formulations of Einstein's field equations. These ideas go beyond casting the evolution equations into symmetric hyperbolic form with maximally dissipative boundary conditions which, except in some rather restricted situations [5, 6, 8, 9], do not seem to be flexible enough to be made compatible with the constraints for metric-based formulations. In particular, we have analysed a gauge condition for the lapse which is obtained by minimizing a functional representing the norm squared of the time derivative of the extrinsic curvature. This leads to a fourth-order elliptic boundary value problem. We have shown that coupling this elliptic problem to the Einstein evolution equations in the $3+1$ decomposition introduced in [22] with a zero shift and imposing constraint-preserving boundary conditions controlling the Weyl scalar $\Psi_{0}$ leads to a well-posed IBVP in the linearized regime. Despite the fact that the results so far have only been obtained in the weak field regime, there are several interesting points on which we would like to comment.

First, obtaining a well-posed problem for linearizations about flat spacetime is a necessary condition for the well posedness of the nonlinear IBVP. This is because the former problem can be obtained from the latter one by considering perturbations with arbitrarily small amplitudes and wavelengths of smooth solutions. There are some problems for which well posedness in the linearized case (together with some technical assumptions) is also sufficient for the well posedness of the nonlinear problem. An example is given by the Cauchy problem for strongly hyperbolic first-order quasi-linear systems. In this case the pseudo-differential calculus allows us to establish well posedness by analysing the equations in the frozen coefficient approximation $[36,37]$. Whether or not these ideas can be generalized to our IBVP remains to be investigated.

Second, it is known that when the lapse is frozen or densitized, the evolution equations we have analysed are only weakly hyperbolic [38, 39]. For such systems there are examples of solutions with frequency-dependent exponentially growing modes [24, 38, 39]. It is therefore a priori not clear that a well-posed IBVP can be obtained for the Einstein evolution equations in [22]. On the other hand, as discussed in section 2, even though these equations are weakly hyperbolic, they induce a 'nice' evolution for the constraint and curvature fields. The wellposed initial-boundary value formulation presented in this paper is based on this particular property of the evolution equations and on the implementation of a fourth-order elliptic gauge condition for the lapse instead of a frozen or densitized lapse. A well-posed elliptichyperbolic formulation for the full nonlinear Einstein equations without boundaries was given in [40].

The next point is related to the discretization of the problem. First, our formulation requires solving a fourth-order elliptic equation at each (or each few) timesteps which might be computationally expensive. In addition to that, since our well posedness proof relies on the propagation of the constraints, it is a priori not clear that a 'naive' discretization of the problem will lead to a stable and convergent scheme. It might be the case that one has to choose very special discretization techniques such that the estimates in the continuum case can be mimicked at the discrete level. This may require some constraint projection mechanism.

Next, we would like to remark that our approach is quite general and should work for any metric formulation of the Einstein equations for which the constraint propagation system can be cast into a symmetric hyperbolic form and for which sufficient regularity for the constraint fields can be shown. In particular, imposing the same gauge condition as in this paper, we expect that a well-posed IBVP can also be derived for families of 
linearized symmetric hyperbolic first-order formulations [41, 42] or linearized mixed firstorder second-order hyperbolic formulations [9, 39] which might be more suitable for numerical discretization.

Finally, the question remains as to what other gauge conditions may work in our approach. In particular, the geometrical meaning of the boundary surface obtained (as embedded in the spacetime constructed) must be clarified. This issue is likely related to the choice of the shift vector. In [31] a functional $J\left(\alpha, \beta^{i}\right)$ of the lapse $\alpha$ and the shift vector $\beta^{i}$ was introduced in order to construct approximate Killing fields for a given data set. For zero shift our functional $I_{G R}[\alpha]$ is closely related to $J$. It should be interesting to investigate gauge conditions that are obtained by varying $J$ with respect to lapse and shift.

These questions, as well as the generalization to linearizations about more general spacetimes including inner excision boundaries, will be considered in future work.

\section{Acknowledgments}

It is a pleasure to thank $\mathrm{H}$ Beyer, G Calabrese, M Holst, L Lehner, H Pfeiffer, O Reula and $\mathrm{M}$ Tiglio for helpful discussions and suggestions and D Reynolds for reading the manuscript. This research was supported in part by NSF grant PHY-0099568, by a grant from the Sherman Fairchild Foundation to Caltech and by NSF DMS Award 0411723 to UCSD.

\section{Appendix A. Compact perturbations of coercive operators}

In this appendix we state the following theorem which is a summary of well-known results.

Theorem 4. Let $X$ be a reflexive Banach space, and let $L: X \rightarrow X^{*}$ be a linear, bounded coercive operator, i.e. $L \in \mathcal{L}\left(X, X^{*}\right)$ and there exists $\delta>0$ such that $L(u)(u) \geqslant \delta\|u\|^{2}$ for all $u \in X$. Furthermore, let $K \in \mathcal{L}\left(X, X^{*}\right)$ be a compact linear operator and set $M:=L+K$. Finally, let $M^{t}: X \rightarrow X^{*}$ denote the bounded linear operator defined by $\left(M^{t} u\right)(v)=(M v)(u)$ for all $u, v \in X$. Then,

(i) $L: X \rightarrow X^{*}$ is invertible with bounded inverse, and $\left\|L^{-1}\right\| \leqslant \delta^{-1}$.

(ii) $\operatorname{ker} M$ and $\operatorname{ker} M^{t}$ are finite dimensional and have equal dimensions.

(iii) $\operatorname{Ran} M=\left(\operatorname{ker} M^{t}\right)^{\perp}$, where $\left(\operatorname{ker} M^{t}\right)^{\perp}$ denotes the annihilator of $\operatorname{ker} M^{t}$.

Proof. Let $I: X \rightarrow X^{* *}$ denote the map defined by $(I u)(\omega)=\omega(u)$ for all $u \in X, \omega \in X^{*}$. Since $X$ is reflexive, $I$ is an isometric isomorphism. Next, let $M^{*} \in \mathcal{L}\left(X^{* *}, X^{*}\right)$ denote the (Banach space) adjoint of $M$, defined by $\left(M^{*} u^{* *}\right)(v)=u^{* *}(M v)$ for all $v \in X, u^{* *} \in X^{* *}$. Then, $M^{t}=M^{*} \circ I$. For the following, we use the formula ${ }^{14}$

$$
\overline{\operatorname{Ran} A}=\left(\operatorname{ker} A^{t}\right)^{\perp},
$$

which holds for any bounded linear operator $A: X \rightarrow X^{*}$ with $A^{t}=A^{*} \circ I$ on a reflexive Banach space $X$.

(i) The coercivity of $L$ implies that

$$
\|L u\| \geqslant \delta\|u\|
$$

and

$$
\left\|L^{t} u\right\| \geqslant \delta\|u\|
$$

${ }^{14}$ See, for instance, [43] section 3 . 
for all $u \in X$. The first inequality implies that $L$ has trivial kernel and closed range, the second inequality implies that $L^{t}$ has trivial kernel. Equation (A.1) then implies that $L$ is bijective. $\left\|L^{-1}\right\| \leqslant \delta^{-1}$ now follows from the inequality (A.2).

(ii) It follows from (i) that $M=L+K$ is Fredholm with the same index as $L$ which is zero $^{15}$. In particular, $M$ has closed range, and $\operatorname{dim} \operatorname{ker} M=\operatorname{dim} \operatorname{ker} M^{t}<\infty$.

(iii) Since $M$ has closed range,

$$
\operatorname{Ran} M=\overline{\operatorname{Ran} M}=\left(\operatorname{ker} M^{t}\right)^{\perp} .
$$

\section{Appendix B. Proof of lemmas 4, 5 and 6}

Proof of lemma 4. (i) Suppose $u_{n}$ is a sequence in $D$ which converges to zero in $X$ and such that $S u_{n}$ converges to $v \in Y$. We have to show that $v=0$. In order to see this, write $u_{n}=\left(\mathbf{h}^{(n)}, \mathbf{k}^{(n)}\right)$ and $v=(H, \mathbf{M})$. Then, $\mathbf{h}^{(n)} \rightarrow 0$ in $H^{1}(\Sigma, S), \mathcal{H} \mathbf{h}^{(n)} \rightarrow H$ in $H^{1}(\Sigma, \mathbb{R})$ and $\mathbf{k}^{(n)} \rightarrow 0$ in $\left.L^{2}(\Sigma, S), \mathcal{M} \mathbf{k}^{(n)}\right] \rightarrow \mathbf{M}$ in $H^{1}(\Sigma, V)$.

Next, take a test function $\varphi \in C_{0}^{\infty}(\Sigma, \mathbb{R})$. Then,

$$
\begin{aligned}
(\varphi, H)_{L^{2}(\Sigma, \mathbb{R})} & =\lim _{n \rightarrow \infty}\left(\varphi, \mathcal{H} \mathbf{h}^{(n)}\right)_{L^{2}(\Sigma, \mathbb{R})} \\
& =\lim _{n \rightarrow \infty} \frac{1}{2}\left(D^{i} \varphi, \delta^{k l} D_{i} h_{k l}^{(n)}-D^{j} h_{i j}^{(n)}\right)_{L^{2}(\Sigma, V)} \\
& =0 .
\end{aligned}
$$

Since this holds for all $\varphi \in C_{0}^{\infty}(\Sigma, \mathbb{R})$ it follows that $H=0$. Similarly, let $\psi \in C_{0}^{\infty}(\Sigma, V)$,

$$
\begin{aligned}
(\psi, \mathbf{M})_{L^{2}(\Sigma, V)} & =\lim _{n \rightarrow \infty}\left(\psi, \mathcal{M} \mathbf{k}^{(n)}\right)_{L^{2}(\Sigma, V)} \\
& =\lim _{n \rightarrow \infty}\left(D^{(i} \psi^{j)}, k_{i j}^{(n)}-\delta_{i j} \delta^{k l} k_{k l}^{(n)}\right)_{L^{2}(\Sigma, S)} \\
& =0
\end{aligned}
$$

which proves that $\mathbf{M}=0$. Therefore, $S_{0}$ is closable. The proof that $T_{0}$ is closable is similar.

(ii) This follows by direct verification.

(iii) Let $u=(\mathbf{h}, \mathbf{k}) \in D_{0}$. Then,

$$
\begin{aligned}
(u, A u)_{X}= & (\mathbf{h},-2 \mathbf{k})_{H^{1}(\Sigma, S)}+\left(\mathbf{k}, P^{(G F)} \operatorname{Ric} \mathbf{h}\right)_{L^{2}(\Sigma, S)} \\
\leqslant & \|\mathbf{h}\|_{H^{1}(\Sigma, S)}^{2}+\|\mathbf{k}\|_{H^{1}(\Sigma, S)}^{2}+\frac{1}{2}\|\mathbf{k}\|_{L^{2}(\Sigma, S)}^{2}+\frac{1}{2}\|\operatorname{Ric} \mathbf{h}\|_{L^{2}(\Sigma, S)}^{2} \\
\leqslant & \|\mathbf{h}\|_{H^{1}(\Sigma, S)}^{2}+C_{1}\left[\|\mathbf{k}\|_{L^{2}(\Sigma, S)}^{2}+\|\mathbf{c u r l} \mathbf{k}\|_{L^{2}(\Sigma, S)}^{2}+\|\mathcal{M} \mathbf{k}\|_{L^{2}(\Sigma, V)}^{2}\right] \\
& +C_{2}\left[\left\|[\operatorname{Ric} \mathbf{h}]^{T F}\right\|_{L^{2}(\Sigma, S)}^{2}+\|\mathcal{H} \mathbf{h}\|_{L^{2}(\Sigma, \mathbb{R})}^{2}\right] \\
\leqslant & a_{0}\|u\|_{X}^{2}+a_{1}\left\|S_{0} u\right\|_{Y}^{2}+a_{2}\left\|T_{0} u\right\|_{Z}^{2}
\end{aligned}
$$

where $C_{1}, C_{2}, a_{0}, a_{1}, a_{2}$ are constants independent of $u$ and where we have used lemma 1 in the third step.

Proof of lemma 5. First, we can assume that $G$ vanishes on one of the boundary components, say on $x=1$. Otherwise, one can construct $\mathbf{h}$ as a superposition of two fields each one satisfying the statement of the lemma for $G$ vanishing on $x=0$ and $x=1$, respectively.

${ }^{15}$ See, for instance, [44], corollaries A.7.2 and A.7.5. 
In order to proceed, let $\psi \in C^{\infty}([0, \infty), \mathbb{R})$ be such that

(i) $\psi^{\prime \prime \prime}(0)=1$,

(ii) $\psi(x)=0$ for all $x \geqslant 2$,

and set $\psi_{n}(x):=n^{-3} \psi(n x)$ for all $n>2$ and $x \in[0,1]$. By construction, $\psi_{n}^{\prime \prime \prime}(0)=1$ and $\psi_{n}(x)=0$ for all $x \in[2 / n, 1]$. Next, define for each $n>2$

$$
h_{i j}^{(n)}(x, y, z)=\left(2 N_{i} N_{j}-\delta_{i j}\right) \psi_{n}(x) G(y, z) .
$$

Since $\psi_{n}^{\prime \prime \prime}(x)=\psi^{\prime \prime \prime}(n x)=0$ for all $x \in[2 / n, 1]$ it follows that

$$
\left\|\mathbf{h}^{(n)}\right\|_{H^{3}(\Sigma, S)}^{2} \leqslant \frac{K}{n}
$$

for a sufficiently large constant $K$ independent of $n$. Therefore, the $H^{3}$-norm of $\mathbf{h}^{(n)}$ can be made arbitrarily small. Furthermore, one finds

$$
\mathcal{H} \mathbf{h}^{(n)}=\frac{1}{n} \psi^{\prime \prime}(n x) G(y, z)
$$

so $\left.N^{i} D_{i}\left(\mathcal{H} \mathbf{h}^{(n)}\right)\right|_{x=0}=G$.

Proof of lemma 6. Similarly to the proof of lemma 5 we may assume that $q$ vanishes on $x=1$. Next, let $\Psi \in C^{\infty}([0, \infty), \mathbb{R})$ be such that

(i) $\Psi(0)=0$,

(ii) $\Psi^{\prime}(0)=1$,

(iii) $\Psi(x)=0$ for all $x \geqslant 2$,

and set $\Psi_{n}(x):=n^{-1} \Psi(n x)$ for all $n>2$ and $x \in[0,1]$. By construction, $\Psi_{n}(0)=$ $0, \Psi_{n}^{\prime}(0)=1$ and $\Psi_{n}(x)=0$ for all $x \in[2 / n, 1]$. Next, define for each $n>2$

$$
k_{k l}^{(n)}(x, y, z)=-\mathrm{i} \Psi_{n}(x) q(y, z) \bar{m}_{k} \bar{m}_{l}+\text { c.c. },
$$

where c.c. denotes the complex conjugate of the previous expression. Since $\Psi_{n}^{\prime}(x)=$ $\Psi^{\prime}(n x)=0$ for all $x \in[2 / n, 1]$ it follows immediately that there is a constant $K>0$ such that

$$
\left\|\mathbf{k}^{(n)}\right\|_{H^{1}(\Sigma, S)}^{2} \leqslant \frac{K}{n}
$$

for all $n>2$. Therefore, the $H^{1}$-norm of $\mathbf{k}^{(n)}$ can be made arbitrarily small. Next, since $\Psi_{n}$ depends on $x$ only one finds

$$
\left(\mathcal{M} \mathbf{k}^{(n)}\right)_{l}=\mathrm{i} \Psi_{n} \bar{m}_{l} \bar{m}^{k} D_{k} q+\text { c.c.. }
$$

Hence $\left.\mathcal{M} \mathbf{k}^{(n)}\right|_{x=0}=0$ and $\left\|\mathcal{M} \mathbf{k}^{(n)}\right\|_{L^{2}(\Sigma, V)}$ can be made arbitrarily small. Finally,

$$
\left(\operatorname{curl~k}{ }^{(n)}\right)_{k l}=\Psi^{\prime}(n x) \bar{m}_{k} \bar{m}_{l} q+\Psi_{n}(x) N_{(k} \bar{m}_{l)} \bar{m}^{j} D_{j} q+\text { c.c. },
$$

which implies that $\left.\left(\operatorname{curl} \mathbf{k}^{(n)}\right)_{k l} m^{k} m^{l}\right|_{x=0} \hat{=} q$.

\section{References}

[1] Friedrich H and Nagy G 1999 The initial boundary value problem for Einstein's vacuum field equations Commun. Math. Phys. 201 619-55

[2] Rauch J 1985 Symmetric positive systems with boundary characteristics of constant multiplicity Trans. Am. Math. Soc. 291 167-87

[3] Secchi P 1996 The initial boundary value problem for linear symmetric hyperbolic systems with characteristic boundary of constant multiplicity Diff. Int. Eqns. 9 671-700 
[4] Secchi P 1996 Well-posedness of characteristic symmetric hyperbolic systems Arch. Ration. Mech. Anal. 134 155-97

[5] Szilagyi B, Schmidt B G and Winicour J 2002 Boundary conditions in linearized harmonic gravity Phys. Rev. D $65064015(1)-064015(15)$

[6] Szilagyi B and Winicour J 2003 Well-posed initial-boundary evolution in general relativity Phys. Rev. D $68041501(1)-041501(5)$

[7] Babiuc M C, Szilagyi B and Winicour J 2006 Harmonic initial-boundary evolution in general relativity Phys. Rev. D 73 064017(1)-064017(23)

[8] Calabrese G, Pullin J, Reula O, Sarbach O and Tiglio M 2003 Well posed constraint-preserving boundary conditions for the linearized Einstein equations Commun. Math. Phys. 240 377-95

[9] Gundlach C and Martín-García J M 2004 Symmetric hyperbolic and consistent boundary conditions for second order Einstein equations Phys. Rev. D 70 044032(1)-044032(16)

[10] Tarfulea N 2005 Constraint preserving boundary conditions for hyperbolic formulations of Einstein's equations PhD Thesis University of Minnesota Preprint gr-qc/0508014

[11] Stewart J M 1998 The Cauchy problem and the initial boundary value problem in numerical relativity Class. Quantum Grav. 15 2865-89

[12] Calabrese G and Sarbach O 2003 Detecting ill posed boundary conditions in general relativity J. Math. Phys. 44 3888-99

[13] Sarbach O and Tiglio M 2005 Boundary conditions for Einstein's field equations: mathematical and numerical analysis J. Hyperbolic Differ. Eqns 2 839-83

[14] Rinne O 2005 Axisymmetric numerical relativity PhD Thesis University of Cambridge Preprint gr-qc/0601064

[15] Calabrese G, Lehner L and Tiglio M 2002 Constraint-preserving boundary conditions in numerical relativity Phys. Rev. D 65 104031(1)-104031(13)

[16] Bardeen J M and Buchman L T 2002 Numerical tests of evolution systems, gauge conditions, and boundary conditions for 1d colliding gravitational plane waves Phys. Rev. D 65 064037(1)-064037(23)

[17] Kidder L E, Lindblom L, Scheel M A, Buchman L and Pfeiffer H P 2005 Boundary conditions for the Einstein evolution system Phys. Rev. D 71 064020(1)-064020(22)

[18] Calabrese G 2003 Constraint preserving boundary conditions for the linearized Einstein equations $P h D$ Thesis Louisiana State University http://etd.lsu.edu/docs/available/etd-1105103-100340

[19] Bona C, Ledvinka T, Palenzuela-Luque C and Zacek M 2005 Constraint-preserving boundary conditions in the Z4 numerical relativity formalism Class. Quantum Grav. 22 2615-34

[20] Lindblom L, Scheel M A, Kidder L E, Owen R and Rinne O 2005 A new generalized harmonic evolution system Preprint gr-qc/0512093

[21] Reula O and Sarbach O 2005 A model problem for the initial-boundary value formulation of Einstein's field equations J. Hyperbolic Differ. Eqns 2 397-435

[22] York J W 1979 Kinematics and dynamics of general relativity Sources of Gravitational Radiation ed L L Smarr (Cambridge: Cambridge University Press) pp 83-126

[23] Nagy G and Sarbach O in preparation

[24] Kreiss H O and Lorenz J 1989 Initial-Boundary Value Problems and the Navier-Stokes Equations (New York: Academic)

[25] Gustafsson B, Kreiss H and Oliger J 1995 Time Dependent Problems and Difference Methods (New York: Wiley)

[26] Calabrese G, Pullin J, Sarbach O and Tiglio M 2002 Convergence and stability in numerical relativity Phys. Rev. D 66 041501(1)-041501(4)

[27] De Donder T 1921 La gravifique einsteinienne (Paris: Gunthier-Villars)

[28] Arnowitt R, Deser S and Misner C 1962 The dynamics of general relativity Gravitation: An Introduction to Current Research ed L Witten (New York: Wiley)

[29] Frittelli S 1997 Note on the propagation of the constraints in standard 3+1 general relativity Phys. Rev. D 55 5992-6

[30] Frittelli S and Gomez R 2003 Einstein boundary conditions for the 3+1 Einstein equations Phys. Rev. D 68 044014(1)-044014(6)

[31] Dain S 2004 A new geometric invariant on initial data for the Einstein equations Phys. Rev. Lett. $93231101(1)-(4)$

[32] Bona C, Lehner L and Palenzuela-Luque C 2005 Geometrically motivated hyperbolic coordinate conditions for numerical relativity: analysis, issues and implementations Phys. Rev. D 72 104009(1)-104009(15)

[33] Ciarlet P G 1997 Mathematical Elasticity, Volume II: Theory of Plates (Amsterdam: Elsevier)

[34] Pazy A 1983 Semigroups of Linear Operators and Applications to Partial Differential Equations (Berlin: Springer) 
[35] Beyer H 2005 Beyond partial differential equations: a course on linear and quasi-linear abstract evolution equations Preprint gr-qc/0510097

[36] Taylor M E 1981 Pseudodifferential Operators (Princeton, NJ: Princeton University Press)

[37] Taylor M E 1991 Pseudodifferential operators and nonlinear PDE Progress in Mathematics vol 100 (Boston, MA: Birkhäuser) (second printing 1993)

[38] Kreiss H O and Ortiz O E 2002 Some mathematical questions connected with first and second order timedependent systems of partial differential equations Lect. Notes Phys. 604 359-70

[39] Nagy G, Ortiz O E and Reula O A 2004 Strongly hyperbolic second order Einstein's evolution equations Phys. Rev. D 70 044012(1)-044012(15)

[40] Andersson L and Moncrief V 2003 Elliptic-hyperbolic systems and the Einstein equations Ann. Henri Poincaré 4 1-34

[41] Frittelli S and Reula O A 1996 First-order symmetric-hyperbolic Einstein equations with arbitrary fixed gauge Phys. Rev. Lett. 76 4667-70

[42] Anderson A and York J W 1999 Fixing Einstein's equations Phys. Rev. Lett. 82 4384-7

[43] Kato T 1980 Perturbation Theory for Linear Operators (New York: Springer)

[44] Taylor M E 1999 Partial Differential Equations, Basic theory (Berlin: Springer) 